\title{
Decuplet baryon masses in covariant baryon chiral perturbation theory
}

\author{
Xiu-Lei Ren, ${ }^{1}$ Li-Sheng Geng, ${ }^{1,2, *}$ and Jie Meng ${ }^{1,3,4}$ \\ ${ }^{1}$ School of Physics and Nuclear Energy Engineering and International \\ Research Center for Nuclei and Particles in the Cosmos, \\ Beihang University, Beijing 100191, China \\ ${ }^{2}$ Physik Department, Technische Universität München, D-85747 Garching, Germany \\ ${ }^{3}$ State Key Laboratory of Nuclear Physics and Technology, \\ School of Physics, Peking University, Beijing 100871, China \\ ${ }^{4}$ Department of Physics, University of Stellenbosch, Stellenbosch 7602, South Africa
}

( $\Omega$ Dated: May 25, 2022)

\begin{abstract}
We present an analysis of the lowest-lying decuplet baryon masses in the covariant baryon chiral perturbation theory with the extended-on-mass-shell scheme up to next-to-next-to-next-to-leading order. In order to determine the 14 low-energy constants, we perform a simultaneous fit of the $n_{f}=2+1$ lattice QCD data from the PACS-CS, QCDSF-UKQCD, and HSC Collaborations, taking finite-volume corrections into account self-consistently. We show that up to next-to-next-to-next-to-leading order one can achieve a good description of the lattice QCD and experimental data. Surprisingly, we note that the current lattice decuplet baryon masses can be fitted rather well by the next-to-leading order baryon chiral perturbation theory, which, however, misses the experimental data a little bit. Furthermore, we predict the pion- and strangeness-sigma terms of the decuplet baryons by use of the Feynman-Hellmann theorem.
\end{abstract}

PACS numbers: 12.39.Fe, 12.38.Gc, 14.20.Gk

Keywords: Chiral Lagrangians, Lattice QCD calculations, Baryon resonances

\footnotetext{
* E-mail me at: lisheng.geng@buaa.edu.cn
} 


\section{INTRODUCTION}

In the past few years many studies of the lowest-lying baryon octet and decuplet masses have been performed on the lattice [1-9] [see Ref. [10] for a comprehensive discussion of the various lattice chromodynamics (LQCD) simulations and the origin of their uncertainties]. These studies not only demonstrate the ability of LQCD simulations to predict accurately nonperturbative observables of the strong interactions, but also provide valuable information that can be used to extract the low-energy constants (LECs) of chiral perturbation theory (ChPT). On the other hand, most LQCD calculations, limited by the availability of computational resources and the efficiency

of algorithms [11], still have to employ larger than physical light-quark masses $m_{u / d}, 1$ finite lattice volume $V=T L^{3}$, and finite lattice spacing $a$. ChPT [15-26] plays an important role in guiding the necessary extrapolations to the physical world in terms of light-quark masses [27-30], lattice volume [31, 32] and lattice spacing [33, 34], and in estimating the induced uncertainties.

As an effective field theory of low-energy QCD, ChPT has been rather successful in the mesonic sector, but the extension to the one-baryon sector turns out to be nontrivial. Because the baryon mass is not zero in the chiral limit, a systematic power counting is absent [18]. In order to restore the chiral power counting, the so-called heavy-baryon (HB) ChPT was first proposed by Jenkins and Manohar [35]. Although this approach provides a strict power counting, the heavy baryon expansion is nonrelativistic. A naive application can lead to pathologies, e.g., in the calculation of the scalar form factor of the nucleon [20].2 In addition, the HB ChPT is found to converge rather slowly in the three-flavor sector of $u, d$, and $s$ quarks. Later, covariant baryon chiral perturbation theory (BChPT) implementing a consistent power counting with different renormalization methods has been developed, such as the infrared (IR) [36] and the extended-on-mass-shell (EOMS) [37, 38] renormalization schemes.

In the past decades, the ground-state octet baryon masses have been studied rather extensively [39-58], especially, in combination with the $n_{f}=2+1$ LQCD data up to next-to-nextto-next-to-leading order ( $\left.\mathrm{N}^{3} \mathrm{LO}\right)$ [50-58]. Different formulations of BChPT have been explored, including the HB ChPT [52], the EOMS BChPT [50, 51, 57, 58], the partial summation approach [53-55], and the IR BChPT [56]. In Refs. [50, 51, 57, 58], we have performed a series of studies on the octet baryon masses by including finite-volume corrections (FVCs) self-consistently

\footnotetext{
${ }^{1}$ It should be noted that for a limited set of observables simulations with physical light-quark masses have recently become available [12-14],

2 This can be removed by resuming the leading kinetic operator to higher orders, equivalent to using the relativistic propagator [36]. 
in the EOMS BChPT up to next-to-next-to-leading order (NNLO) and $\mathrm{N}^{3} \mathrm{LO}$. In these studies, we found that the $\mathrm{N}^{3} \mathrm{LO}$ EOMS BChPT can provide a good description of all the current LQCD data for the octet baryon masses, and we confirmed that the LQCD results are consistent with each other, although their setups are different. Furthermore, the FVCs to the LQCD data are found to be important not only for the purpose of chiral extrapolations, but also for the determination of the corresponding LECs, especially for the many LECs appearing at $\mathrm{N}^{3} \mathrm{LO}$.

On the contrary, there are only a few studies of the $n_{f}=2+1$ LQCD decuplet baryon masses [5, 53-55, 59]. In Refs. [5, 59], it was shown that the HB ChPT at NNLO cannot describe the LHPC and PACS-CS octet and decuplet baryon masses. This has motivated the series of studies on the $n_{f}=2+1$ LQCD octet baryon masses in the EOMS framework [50, 51, 57, 58]. The PACS-CS and LHPC decuplet baryon data were also studied in Ref. [50] up to NNLO and a reasonable description of the LQCD data was achieved, contrary to the HB ChPT studies of Refs. [5, 59]. In Ref. [53], Semke and Lutz studied the BMW [1] LQCD data for the octet and decuplet baryon masses up to $\mathrm{N}^{3} \mathrm{LO}$ in BChPT with the partial summation scheme. It was shown that the lightquark mass dependence of the decuplet baryon masses can be well described. However, FVCs to the lattice data are not taken into account self-consistently. Whereas it has been shown in Refs. [51, 57, 58] that FVCs need to be taken into account self-consistently in order to achieve a $\chi^{2} /$ d.o.f. of about 1 in the description of the current $n_{f}=2+1$ LQCD octet baryon masses.

Given the fact that a simultaneous description of the $n_{f}=2+1$ LQCD decuplet baryon masses with FVCs taken into account self-consistently is still missing and that the EOMS BChPT can describe the LQCD octet baryon masses rather well [50, 51, 57, 58], it is timely to perform a thorough study of the lowest-lying decuplet baryon masses in the EOMS BChPT up to $\mathrm{N}^{3} \mathrm{LO}$. The paper is organized as follows: In Sec. II, we collect the relevant chiral effective Lagrangians, which contain 14 to be determined LECs, and calculate the decuplet baryon masses and the corresponding FVCs in covariant BChPT up to $\mathrm{N}^{3} \mathrm{LO}$. In Sec. III, we perform a simultaneous fit of the PACS-CS, QCDSF-UKQCD, and HSC data, study the convergence of BChPT and the contributions of virtual octet and decuplet baryons, and compare the $\mathrm{N}^{3} \mathrm{LO}$ BChPT with those LQCD data not included in the fit. We then predict the pion- and strangeness-baryon sigma terms with the LECs determined from the best fits and compare them with the results of other recent studies. A short summary is given in Sec. IV 


\section{THEORETICAL FRAMEWORK}

\section{A. Chiral effective Lagrangians}

The chiral effective Lagrangians relevant to the present study can be written as the sum of a mesonic part and a meson-baryon part:

$$
\mathcal{L}_{\text {eff }}=\mathcal{L}_{\phi}^{(2)}+\mathcal{L}_{\phi}^{(4)}+\mathcal{L}_{\phi D}^{(1)}+\mathcal{L}_{\phi D}^{(2)}+\mathcal{L}_{\phi D}^{(4)}
$$

The Lagrangians $\mathcal{L}_{\phi}^{(2)}$ and $\mathcal{L}_{\phi}^{(4)}$ of the mesonic sector can be found in Ref. [57]. The leading order meson-baryon Lagrangian is

$$
\mathcal{L}_{\phi D}^{(1)}=\mathcal{L}_{D}+\mathcal{L}_{\phi B D}^{(1)}+\mathcal{L}_{\phi D D}^{(1)}
$$

where $\mathcal{L}_{D}$ denotes the covariant free Lagrangian, and $\mathcal{L}_{\phi B D}^{(1)}$ and $\mathcal{L}_{\phi D D}^{(1)}$ describe the interaction of the octet and decuplet baryons with the pseudoscalar mesons and have the following form:

$$
\begin{aligned}
\mathcal{L}_{D} & =\bar{T}_{\mu}^{a b c}\left(i \gamma^{\mu \nu \alpha} D_{\alpha}-m_{D} \gamma^{\mu \nu}\right) T_{v}^{a b c}, \\
\mathcal{L}_{\phi B D}^{(1)} & =\frac{i C}{m_{D} F_{\phi}} \varepsilon^{a b c}\left(\partial_{\alpha} \bar{T}_{\mu}^{a d e}\right) \gamma^{\alpha \mu \nu} B_{c}^{e} \partial_{\nu} \phi_{b}^{d}+\text { H.c. }, \\
\mathcal{L}_{\phi D D}^{(1)} & =\frac{i \mathcal{H}}{m_{D} F_{\phi}} \bar{T}_{\mu}^{a b c} \gamma^{\mu \nu \rho \sigma} \gamma_{5}\left(\partial_{\rho} T_{v}^{a b d}\right) \partial_{\sigma} \phi_{d}^{c},
\end{aligned}
$$

where we have used the so-called "consistent" coupling scheme for the meson-octet-decuplet vertices [60,61]. In the above Lagrangians, $m_{D}$ is the decuplet baryon mass in the chiral limit and $T$ is the decuplet baryon field represented by the Rarita-Schwinger field $T^{a b c} \equiv T_{\mu}^{a b c}$. The physical fields are assigned as $T^{111}=\Delta^{++}, T^{112}=\Delta^{+} / \sqrt{3}, T^{122}=\Delta^{0} / \sqrt{3}, T^{222}=\Delta^{-}, T^{113}=\Sigma^{*+} / \sqrt{3}$, $T^{123}=\Sigma^{* 0} / \sqrt{6}, T^{223}=\Sigma^{*-} / \sqrt{3}, T^{133}=\Xi^{* 0} / \sqrt{3}, T^{233}=\Xi^{*-} / \sqrt{3}$, and $T^{333}=\Omega^{-} . D_{v} T_{\mu}^{a b c}=$ $\partial_{\nu} T_{\mu}^{a b c}+\left(\Gamma_{v}, T_{\mu}\right)^{a b c}, \Gamma_{v}=\frac{1}{2}\left\{u^{\dagger} \partial_{\nu} u+u \partial_{\nu} u^{\dagger}\right\}$ being the chiral connection with $u=\exp \left(i \frac{\phi}{2 F_{\phi}}\right)$ collecting the pseudoscalar fields $\phi$, and $\left(X, T_{\mu}\right)^{a b c} \equiv(X)_{d}^{a} T_{\mu}^{d b c}+(X)_{d}^{b} T_{\mu}^{a d c}+(X)_{d}^{c} T_{\mu}^{a b d}$. The coefficient $F_{\phi}$ is the meson-decay constant in the chiral limit, and $C(\mathcal{H})$ denotes the $\phi B D(\phi D D)$ coupling. The totally antisymmetric gamma matrix products are defined as $\gamma^{\mu \nu}=\frac{1}{2}\left[\gamma^{\mu}, \gamma^{\nu}\right]$, $\gamma^{\mu \nu \rho}=\frac{1}{2}\left\{\gamma^{\mu \nu}, \gamma^{\rho}\right\}=-i \varepsilon^{\mu \nu \rho \sigma} \gamma_{\sigma} \gamma_{5}, \gamma^{\mu \nu \rho \sigma}=\frac{1}{2}\left[\gamma^{\mu \nu \rho}, \gamma^{\sigma}\right]=i \varepsilon^{\mu \nu \rho \sigma} \gamma_{5}$ with the following conventions: $g^{\mu \nu}=\operatorname{diag}(1,-1,-1,-1), \varepsilon_{0,1,2,3}=-\varepsilon^{0,1,2,3}=1, \gamma_{5}=i \gamma_{0} \gamma_{1} \gamma_{2} \gamma_{3}$ [62]. In the last and following Lagrangians, we sum over any repeated $\mathrm{SU}(3)$ index denoted by latin characters $a, b, c, \ldots$, and $(X)_{b}^{a}$ denotes the element of row $a$ and column $b$ of the matrix representation of $X$.

The meson-baryon Lagrangian at order $O\left(p^{2}\right)$ can be written as

$$
\mathcal{L}_{\phi D}^{(2)}=\mathcal{L}_{\phi B}^{(2, \mathrm{sb})}+\mathcal{L}_{\phi D}^{(2, \mathrm{sb})}+\mathcal{L}_{\phi D}^{(2) '}
$$


The first and second terms denote the explicit chiral symmetry breaking part:

$$
\begin{aligned}
& \mathcal{L}_{\phi B}^{(2, \mathrm{sb})}=b_{0}\langle\bar{B} B\rangle\left\langle\chi_{+}\right\rangle+b_{D / F}\left\langle\bar{B}\left[\chi_{+}, B\right]_{ \pm}\right\rangle, \\
& \mathcal{L}_{\phi D}^{(2, \mathrm{sb})}=\frac{t_{0}}{2} \bar{T}_{\mu}^{a b c} g^{\mu \nu} T_{v}^{a b c}\left\langle\chi_{+}\right\rangle+\frac{t_{D}}{2} \bar{T}_{\mu}^{a b c} g^{\mu \nu}\left(\chi_{+}, T_{v}\right)^{a b c},
\end{aligned}
$$

where $b_{0}, b_{D}, b_{F}, t_{0}$, and $t_{D}$ are the LECs, $\chi_{+}=u^{\dagger} \chi u^{\dagger}+u \chi^{\dagger} u$, and $\chi=2 B_{0} \mathcal{M}$ accounts for explicit chiral symmetry breaking with $B_{0}=-\langle 0|\bar{q} q| 0\rangle / F_{\phi}^{2}$ and $\mathcal{M}=\operatorname{diag}\left(m_{l}, m_{l}, m_{s}\right)$. For the chiral symmetry conserving part $\mathcal{L}_{\phi D}^{(2)^{\prime}}$, one has nine terms, following the conventions of Refs. [53, 63],

$$
\begin{aligned}
\mathcal{L}_{\phi D}^{(2) \prime}= & \frac{1}{F_{\phi}^{2}}\left\{t_{1} \bar{T}_{\mu}^{a b c} g^{\mu \nu}\left(\partial^{\sigma} \phi \partial_{\sigma} \phi\right)_{c}^{d} T_{v}^{a b d}+t_{2} \bar{T}_{\mu}^{a b c}\left[\left(\partial^{\mu} \phi \partial_{\nu} \phi\right)_{c}^{d}+\left(\partial_{\nu} \phi \partial^{\mu} \phi\right)_{c}^{d}\right] T^{\nu, a b d}\right. \\
& +t_{3} \bar{T}_{\mu, a b c} \partial_{\nu} \phi_{d}^{a} \varepsilon^{b d e} T^{\mu, f g c} \partial^{v} \phi_{f}^{h} \varepsilon_{g h e}+t_{4} \bar{T}_{\mu, a b c}\left[\partial^{\mu} \phi_{d}^{a} \varepsilon^{b d e} \partial_{\nu} \phi_{f}^{h} \varepsilon_{g h e}+\partial_{\nu} \phi_{d}^{a} \varepsilon^{b d e} \partial^{\mu} \phi_{f}^{h} \varepsilon_{g h e}\right] T^{\nu, f g c} \\
& +t_{5} \bar{T}_{\mu}^{a b c} g^{\mu \nu} T_{v}^{a b c}\left\langle\partial^{\sigma} \phi \partial_{\sigma} \phi\right\rangle+t_{6} \bar{T}_{\mu}^{a b c} T^{\nu, a b c}\left\langle\partial^{\mu} \phi \partial_{\nu} \phi\right\rangle \\
& +t_{7}\left[\left(\bar{T}_{\alpha}^{a b c}\left(\partial_{\mu} \phi \partial_{\nu} \phi\right)_{c}^{d} i \gamma^{\mu} \partial^{\nu} T^{\alpha, a b d}+\bar{T}_{\alpha}^{a b c}\left(\partial_{\nu} \phi \partial_{\mu} \phi\right)_{c}^{d} i \gamma^{\mu} \partial^{v} T^{\alpha, a b d}\right)+\text { H.c. }\right] \\
& +t_{8}\left[\left(\bar{T}_{\alpha, a b c} \partial_{\mu} \phi_{d}^{a} \varepsilon^{b d e} i \gamma^{\mu} \partial^{v} T^{\alpha, f g c} \partial_{\nu} \phi_{f}^{h} \varepsilon_{g h e}+\bar{T}_{\alpha, a b c} \partial_{\nu} \phi_{d}^{a} \varepsilon^{b d e} i \gamma^{\mu} \partial^{v} T^{\alpha, f g c} \partial_{\mu} \phi_{f}^{h} \varepsilon_{g h e}\right)+\text { H.c. }\right] \\
& \left.+t_{9}\left[\bar{T}_{\alpha}^{a b c} i \gamma^{\mu} \partial^{\nu} T^{\alpha, a b c}\left\langle\partial_{\mu} \phi \partial_{\nu} \phi\right\rangle+\text { H.c. }\right]\right\},
\end{aligned}
$$

where $t_{1, \ldots, 6}$ have dimension mass ${ }^{-1}$ and $t_{7, \ldots, 9}$ have dimension mass ${ }^{-2}$.

The fourth order chiral effective Lagrangians contain five LECs (see also Refs. [53, 64]):

$$
\begin{aligned}
\mathcal{L}_{\phi D}^{(4)}= & e_{1} \bar{T}_{\mu}^{a b c} g^{\mu v}\left(\chi_{+}^{2}\right)_{d}^{c} T_{v}^{a b d}+e_{2}\left(\bar{T}_{\mu}^{a b c}\left(\chi_{+}\right)_{c}^{d}\right) g^{\mu v}\left(\left(\chi_{+}\right)_{e}^{b} T_{v}^{a e d}\right) \\
& +e_{3} \bar{T}_{\mu}^{a b c} g^{\mu v}\left(\chi_{+}\right)_{d}^{c} T_{v}^{a b d}\left\langle\chi_{+}\right\rangle+e_{4} \bar{T}_{\mu}^{a b c} g^{\mu v} T_{v}^{a b c}\left\langle\chi_{+}\right\rangle^{2} \\
& +e_{5} \bar{T}_{\mu}^{a b c} g^{\mu v} T_{v}^{a b c}\left\langle\chi_{+}^{2}\right\rangle .
\end{aligned}
$$

The propagator of the spin-3/2 fields in $d$ dimensions has the following form [65]:

$$
S^{\mu \nu}(p)=-\frac{\not p+m_{D}}{p^{2}-m_{D}^{2}+i \epsilon}\left[g^{\mu v}-\frac{1}{d-1} \gamma^{\mu} \gamma^{v}-\frac{1}{(d-1) m_{D}}\left(\gamma^{\mu} p^{v}-\gamma^{v} p^{\mu}\right)-\frac{d-2}{(d-1) m_{D}^{2}} p^{\mu} p^{v}\right] .
$$

\section{B. Decuplet baryon masses}

In this subsection, the decuplet baryon masses are calculated in the limit of exact isospin symmetry. Formally, up to $O\left(p^{4}\right)$ the baryon masses can be written as

$$
M_{D}=m_{D}+m_{D}^{(2)}+m_{D}^{(3)}+m_{D}^{(4)}
$$




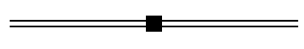

(a)

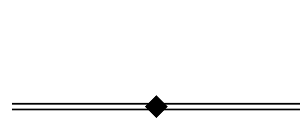

(d)

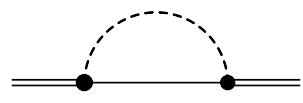

(b)

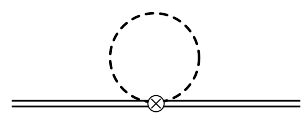

(e)

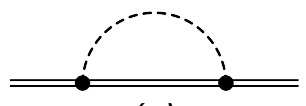

(c)

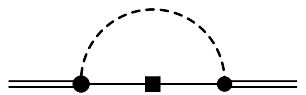

(f)

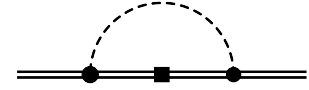

(g)

FIG. 1. Feynman diagrams contributing to the decuplet baryon masses up to $O\left(p^{4}\right)$ in the EOMS BChPT. The single lines correspond to octet baryons, double lines to decuplet baryons and dashed lines to mesons. The black boxes (diamond) indicate second (fourth) order couplings. The solid dot (circle-cross) indicates an insertion from the dimension one (two) meson-baryon Lagrangians. Wave function renormalization diagrams are not explicitly shown but included in the calculation.

where $m_{D}$ is the decuplet baryon mass in the chiral limit. The $m_{D}^{(2)}, m_{D}^{(3)}$, and $m_{D}^{(4)}$ are the next-toleading order (NLO), NNLO, and $\mathrm{N}^{3} \mathrm{LO}$ chiral corrections to the decuplet baryon masses, respectively. The corresponding Feynman diagrams are shown in Fig. 1, and the explicit expression of the decuplet baryon masses is

$$
\begin{aligned}
M_{D}= & m_{D}+\xi_{D, \pi}^{(a)} M_{\pi}^{2}+\xi_{D, K}^{(a)} M_{K}^{2} \\
& +\frac{1}{\left(4 \pi F_{\phi}\right)^{2}} \sum_{\phi=\pi, K, \eta}\left[\xi_{D, \phi}^{(b)} H_{D}^{(b)}\left(M_{\phi}\right)+\xi_{D, \phi}^{(c)} H_{D}^{(c)}\left(M_{\phi}\right)\right] \\
& +\xi_{D, \pi}^{(d)} M_{\pi}^{4}+\xi_{D, K}^{(d)} M_{K}^{4}+\xi_{D, \pi K}^{(d)} M_{\pi}^{2} M_{K}^{2} \\
& +\frac{1}{\left(4 \pi F_{\phi}\right)^{2}} \sum_{\phi=\pi, K, \eta}\left[\xi_{D, \phi}^{(e, 1)} H_{D}^{(e, 1)}\left(M_{\phi}\right)+\xi_{D, \phi}^{(e, 2)} H_{D}^{(e, 2)}\left(M_{\phi}\right)+\xi_{D, \phi}^{(e, 3)} H_{D}^{(e, 3)}\left(M_{\phi}\right)\right] \\
& -\frac{1}{\left(4 \pi F_{\phi}\right)^{2}} \sum_{\substack{\phi=\pi, K, \eta \\
B=N, \Lambda, \Sigma, \Xi}} \xi_{D B, \phi}^{(f)} H_{D, B}^{(f)}\left(M_{\phi}\right) \\
& -\frac{1}{\left(4 \pi F_{\phi}\right)^{2}} \sum_{\substack{\phi=\pi, K, \eta \\
D^{\prime}=\Delta, \Sigma^{*}, \Xi^{*}, \Omega^{-}}} \xi_{D D^{\prime}, \phi}^{(g)} H_{D, D^{\prime}}^{(g)}\left(M_{\phi}\right),
\end{aligned}
$$

where $\xi^{(i)}$ 's and $H^{(i)}$ 's are the corresponding coefficients and loop functions with the subscript $i$ denoting the corresponding diagrams shown in Fig. 1. The $\xi^{(i)}$ 's are tabulated in Tables $\llbracket$ and

In Eq. (13), the loop functions $H_{D}^{(b)}, H_{D}^{(c)}, H_{D}^{(e, 1)}, H_{D}^{(e, 2)}, H_{D}^{(e, 3)}, H_{D, B}^{(f)}$, and $H_{D, D^{\prime}}^{(g)}$ are obtained by using the $\overline{\mathrm{MS}}$ renormalization scheme to remove the divergent pieces and the EOMS renormalization scheme to remove the power-counting-breaking (PCB) terms [37, 38, 66]. The explicit expressions of $H_{D}^{(b)}, H_{D}^{(c)}$ can be found in Ref. [50], and the others are given in the Appendix.

It should be noted that in the evaluation of the diagrams in Figs. 1(f) and (g), we have only kept 
TABLE I. Coefficients of the NLO and NNLO contributions to the decuplet baryon masses [Eq. [13)].

\begin{tabular}{ccccc}
\hline \hline & $\Delta$ & $\Sigma^{*}$ & $\Xi^{*}$ & $\Omega^{-}$ \\
\hline$\xi_{D, \pi}^{(a)}$ & $t_{0}+3 t_{D}$ & $t_{0}+t_{D}$ & $t_{0}-t_{D}$ & $t_{0}-3 t_{D}$ \\
$\xi_{D, K}^{(a)}$ & $2 t_{0}$ & $2 t_{0}+2 t_{D}$ & $2 t_{0}+4 t_{D}$ & $2 t_{0}+6 t_{D}$ \\
\hline$\xi_{D, \pi}^{(b)}$ & $\frac{4}{3} C^{2}$ & $\frac{10}{9} C^{2}$ & $\frac{2}{3} C^{2}$ & 0 \\
$\xi_{D, K}^{(b)}$ & $\frac{4}{3} C^{2}$ & $\frac{8}{9} C^{2}$ & $\frac{4}{3} C^{2}$ & $\frac{8}{3} C^{2}$ \\
$\xi_{D, \eta}^{(b)}$ & 0 & $\frac{2}{3} C^{2}$ & $\frac{2}{3} C^{2}$ & 0 \\
\hline$\xi_{D, \pi}^{(c)}$ & $\frac{50}{27} \mathcal{H}^{2}$ & $\frac{80}{81} \mathcal{H}^{2}$ & $\frac{10}{27} \mathcal{H}^{2}$ & 0 \\
$\xi_{D, K}^{(c)}$ & $\frac{20}{27} \mathcal{H}^{2}$ & $\frac{160}{81} \mathcal{H}^{2}$ & $\frac{20}{9} \mathcal{H}^{2}$ & $\frac{40}{27} \mathcal{H}^{2}$ \\
$\xi_{D, \eta}^{(c)}$ & $\frac{10}{27} \mathcal{H}^{2}$ & 0 & $\frac{10}{27} \mathcal{H}^{2}$ & $\frac{40}{27} \mathcal{H}^{2}$ \\
\hline \hline
\end{tabular}

terms linear in $M_{D}^{(2)}$ and $M_{B}^{(2)}$, in accordance with our power counting. At $\mathrm{N}^{3} \mathrm{LO}$, the pseudoscalar meson masses appearing in $m_{D}^{(2)}$ should be replaced by their $O\left(p^{4}\right)$ counterparts to generate the $\mathrm{N}^{3} \mathrm{LO}$ contributions to $m_{D}^{(4)}$. The explicit expressions of the meson masses up to $O\left(p^{4}\right)$ can be found in Ref. [17]. The empirical values of the LECs $L_{i}^{r}(i=1, \ldots, 10)$ are taken from the latest global fit [67]. In order to be consistent with our renormalization scale used for the baryon sector, we have reevaluated the LECs at $\mu=1 \mathrm{GeV}$. The details can be found in Ref. [57]. 3

\section{Finite-volume corrections}

As emphasized in Refs. [51, 57, 58], FVCs have to be taken into account in studying the current LQCD data. In the case of the decuplet baryon masses, they have been studied up to NNLO in the EOMS BChPT [50] and in the HB ChPT [59]. In the following, we extend the study up to $\mathrm{N}^{3} \mathrm{LO}$ in the EOMS BChPT.

The FVCs can be easily evaluated following the standard technique. One chooses the baryon rest frame, i.e., $p^{\mu}=\left(m_{D}, \overrightarrow{0}\right)$, performs a momentum shift and wick rotation, integrates over the temporal dimension, and obtains the results expressed in terms of the master formulas given in Ref. [32]. See Refs.[31, 32, 51] for more details.

\footnotetext{
${ }^{3}$ In both Ref. [57] and the present work, the LQCD pseudoscalar masses are treated as LO masses. We have checked that treating them as NLO masses does not affect in any significant way the results of both studies.
} 
TABLE II. Coefficients of the $\mathrm{N}^{3} \mathrm{LO}$ contributions to the decuplet baryon masses [Eq. (13)], with the following notations: $\tilde{t}_{1}=2 t_{1}+t_{2}, \tilde{t}_{2}=2 t_{3}+t_{4}$, and $\tilde{t}_{3}=4 t_{5}+t_{6}$.

\begin{tabular}{|c|c|c|c|c|}
\hline & $\Delta$ & $\Sigma^{*}$ & $\Xi^{*}$ & $\Omega^{-}$ \\
\hline$\xi_{D, \pi}^{(d)}$ & $4\left(e_{1}+e_{2}+e_{3}+e_{4}+3 e_{5}\right)$ & $\frac{4}{3}\left(3 e_{1}-e_{2}+e_{3}+3 e_{4}+9 e_{5}\right)$ & $\frac{4}{3}\left(3 e_{1}-e_{2}-e_{3}+3 e_{4}+9 e_{5}\right)$ & $4\left(e_{1}+e_{2}-e_{3}+e_{4}+3 e_{5}\right)$ \\
\hline$\xi_{D, K}^{(d)}$ & $16\left(e_{4}+e_{5}\right)$ & $\frac{16}{3}\left(e_{1}+e_{3}+3 e_{4}+3 e_{5}\right)$ & $\frac{16}{3}\left(2 e_{1}+e_{2}+2 e_{3}+3 e_{4}+3 e_{5}\right)$ & $16\left(e_{1}+e_{2}+e_{3}+e_{4}+e_{5}\right)$ \\
\hline$\xi_{D, \pi K}^{(d)}$ & $8\left(e_{3}+2 e_{4}-2 e_{5}\right)$ & $-\frac{16}{3}\left(e_{1}-e_{2}-e_{3}-3 e_{4}+3 e_{5}\right)$ & $-\frac{8}{3}\left(4 e_{1}-e_{3}-6 e_{4}+6 e_{5}\right)$ & $-16\left(e_{1}+e_{2}-e_{4}+e_{5}\right)$ \\
\hline$\xi_{D, \pi}^{(e, 1)}$ & $\frac{3}{2}\left(2 t_{0}+3 t_{D}\right) M_{\pi}^{2}$ & $3\left(t_{0}+t_{D}\right) M_{\pi}^{2}$ & $\frac{3}{2}\left(2 t_{0}+t_{D}\right) M_{\pi}^{2}$ & $3 t_{0} M_{\pi}^{2}$ \\
\hline$\xi_{D, K}^{(e, 1)}$ & $\left(4 t_{0}+3 t_{D}\right) M_{K}^{2}$ & $4\left(t_{0}+t_{D}\right) M_{K}^{2}$ & $\left(4 t_{0}+5 t_{D}\right) M_{K}^{2}$ & $2\left(2 t_{0}+3 t_{D}\right) M_{K}^{2}$ \\
\hline$\xi_{D, \eta}^{(e, 1)}$ & $\frac{1}{6}\left[8 t_{0} M_{K}^{2}-\left(2 t_{0}-3 t_{D}\right) M_{\pi}^{2}\right]$ & $\frac{1}{3}\left(t_{0}+t_{D}\right)\left(4 M_{K}^{2}-M_{\pi}^{2}\right)$ & $\frac{1}{6}\left[8\left(t_{0}+2 t_{D}\right) M_{K}^{2}-\left(2 t_{0}+7 t_{D}\right) M_{\pi}^{2}\right]$ & $\frac{1}{3}\left[4\left(t_{0}+3 t_{D}\right) M_{K}^{2}-\left(t_{0}+6 t_{D}\right) M_{\pi}^{2}\right]$ \\
\hline$\xi_{D, \pi}^{(e, 2)}$ & $-\frac{1}{2}\left(3 \tilde{t}_{1}+2 \tilde{t}_{2}+3 \tilde{t}_{3}\right)$ & $-\frac{1}{6}\left(6 \tilde{t}_{1}+5 \tilde{t}_{2}+9 \tilde{t}_{3}\right)$ & $-\frac{1}{2}\left(\tilde{t}_{1}+\tilde{t}_{2}+3 \tilde{t}_{3}\right)$ & $-\frac{3}{2} \tilde{t}_{3}$ \\
\hline$\xi_{D, K}^{(e, 2)}$ & $-\left(\tilde{t}_{1}+\tilde{t}_{2}+2 \tilde{t}_{3}\right)$ & $-\frac{2}{3}\left(2 \tilde{t}_{1}+\tilde{t}_{2}+3 \tilde{t}_{3}\right)$ & $-\frac{1}{3}\left(5 \tilde{t}_{1}+3 \tilde{t}_{2}+6 \tilde{t}_{3}\right)$ & $-2\left(\tilde{t}_{1}+\tilde{t}_{2}+\tilde{t}_{3}\right)$ \\
\hline$\xi_{D, \eta}^{(e, 2)}$ & $-\frac{1}{6}\left(\tilde{t}_{1}+3 \tilde{t}_{3}\right)$ & $-\frac{1}{6}\left(2 \tilde{t}_{1}+3 \tilde{t}_{2}+3 \tilde{t}_{3}\right)$ & $-\frac{1}{2}\left(\tilde{t}_{1}+\tilde{t}_{2}+\tilde{t}_{3}\right)$ & $-\frac{1}{6}\left(4 \tilde{t}_{1}+3 \tilde{t}_{3}\right)$ \\
\hline$\xi_{D, \pi}^{(e, 3)}$ & $-4\left(3 t_{7}+2 t_{8}+3 t_{9}\right)$ & $-\frac{4}{3}\left(6 t_{7}+5 t_{8}+9 t_{9}\right)$ & $-4\left(t_{7}+t_{8}+3 t_{9}\right)$ & $-12 t_{9}$ \\
\hline$\xi_{D, K}^{(e, 3)}$ & $-8\left(t_{7}+t_{8}+2 t_{9}\right)$ & $-\frac{16}{3}\left(2 t_{7}+t_{8}+3 t_{9}\right)$ & $-\frac{8}{3}\left(5 t_{7}+3 t_{8}+6 t_{9}\right)$ & $-16\left(t_{7}+t_{8}+t_{9}\right)$ \\
\hline$\xi_{D, \eta}^{(e, 3)}$ & $-\frac{4}{3}\left(t_{7}+3 t_{9}\right)$ & $-\frac{4}{3}\left(2 t_{7}+3 t_{8}+3 t_{9}\right)$ & $-4\left(t_{7}+t_{8}+t_{9}\right)$ & $-\frac{4}{3}\left(4 t_{7}+3 t_{9}\right)$ \\
\hline$\xi_{D N,\{\pi, K, \eta\}}^{(f)}$ & $\left\{2 C^{2}, 0,0\right\}$ & $\left\{0, \frac{2}{3} C^{2}, 0\right\}$ & $\{0,0,0\}$ & $\{0,0,0\}$ \\
\hline$\xi_{D \Lambda,\{\pi, K, \eta\}}^{(f)}$ & $\{0,0,0\}$ & $\left\{C^{2}, 0,0\right\}$ & $\left\{0, C^{2}, 0\right\}$ & $\{0,0,0\}$ \\
\hline$\xi_{D \Sigma,\{\pi, K, \eta\}}^{(f)}$ & $\left\{0,2 C^{2}, 0\right\}$ & $\left\{\frac{2}{3} C^{2}, 0, C^{2}\right\}$ & $\left\{0, C^{2}, 0\right\}$ & $\{0,0,0\}$ \\
\hline$\xi_{D \Xi,\{\pi, K, \eta\}}^{(f)}$ & $\{0,0,0\}$ & $\left\{0, \frac{2}{3} C^{2}, 0\right\}$ & $\left\{C^{2}, 0, C^{2}\right\}$ & $\left\{0,0,4 C^{2}\right\}$ \\
\hline$\xi_{D \Delta,\{\pi, K, \eta\}}^{(g)}$ & $\left\{\frac{5}{3} \mathcal{H}^{2}, 0, \frac{1}{3} \mathcal{H}^{2}\right\}$ & $\left\{0, \frac{8}{9} \mathcal{H}^{2}, 0\right\}$ & $\{0,0,0\}$ & $\{0,0,0\}$ \\
\hline$\xi_{D \Sigma^{*},\{\pi, K, \eta\}}^{(g)}$ & $\left\{0, \frac{2}{3} \mathcal{H}^{2}, 0\right\}$ & $\left\{\frac{8}{9} \mathcal{H}^{2}, 0,0\right\}$ & $\left\{0, \frac{4}{3} \mathcal{H}^{2}, 0\right\}$ & $\{0,0,0\}$ \\
\hline$\xi_{D \Xi^{*},\{\pi, K, \eta\}}^{(g)}$ & $\{0,0,0\}$ & $\left\{0, \frac{8}{9} \mathcal{H}^{2}, 0\right\}$ & $\left\{\frac{1}{3} \mathcal{H}^{2}, 0, \frac{1}{3} \mathcal{H}^{2}\right\}$ & $\left\{0, \frac{4}{3} \mathcal{H}^{2}, 0\right\}$ \\
\hline$\xi_{D \Omega^{-},\{\pi, K, \eta\}}^{(g)}$ & $\{0,0,0\}$ & $\{0,0,0\}$ & $\left\{0, \frac{2}{3} \mathcal{H}^{2}, 0\right\}$ & $\left\{0,0, \frac{4}{3} \mathcal{H}^{2}\right\}$ \\
\hline
\end{tabular}

To proceed with the above procedure, one should note that since Lorentz invariance is lost in finite volume, the mass term in the loop functions is identified as the term having the structure of $\delta_{i j}$. This can be easily seen by noticing that at the rest frame the zero component of the decuplet baryon field vanishes because of the on-shell condition $p_{\mu} T^{\mu}=0$. For instance, the loop function of the diagram in Fig. 1(b), after Feynman parametrization, becomes

$$
G_{D}^{(b)} \propto \int \frac{d^{4} k}{(2 \pi)^{4}} \frac{\left(m_{D}(x-1)-m_{B}\right) k^{\alpha} k^{\beta}}{\left(k^{2}-\mathcal{M}_{D}^{(b)^{2}}\right)^{2}}
$$

where $\mathcal{M}_{D}^{(b)^{2}}=\left(x^{2}-x\right) m_{D}^{2}+x m_{0}^{2}+(1-x) M_{\phi}^{2}-i \epsilon$. To evaluate its contribution to the decuplet baryon mass, one simply replaces $k^{\alpha} k^{\beta}$ with $\delta_{i j} \vec{k}^{2} / 3$ in the numerator. Following the procedure specified 
above, one can then easily obtain the FVCs to the loop function of the diagram in Fig. 1(b),

$$
\begin{aligned}
\delta G_{D}^{(b)}\left(M_{\phi}\right) & \equiv G_{D}^{(b)}(L)-G_{D}^{(b)}(\infty) \\
& =-\frac{1}{12} \int_{0}^{1} d x\left[m_{0}-m_{D}(x-1)\right]\left[\delta_{1 / 2}\left(\mathcal{M}_{D}^{(b)^{2}}\right)-\mathcal{M}_{D}^{(b)^{2}} \delta_{3 / 2}\left(\mathcal{M}_{D}^{(b)^{2}}\right)\right],
\end{aligned}
$$

where the "master" formulas $\delta_{r}\left(\mathcal{M}^{2}\right)$ are defined as

$$
\delta_{r}\left(\mathcal{M}^{2}\right)=\frac{2^{-1 / 2-r}\left(\sqrt{\mathcal{M}^{2}}\right)^{3-2 r}}{\pi^{3 / 2} \Gamma(r)} \sum_{\vec{n} \neq 0}\left(L \sqrt{\mathcal{M}^{2}}|\vec{n}|\right)^{-3 / 2+r} K_{3 / 2-r}\left(L \sqrt{\mathcal{M}^{2}}|\vec{n}|\right),
$$

where $K_{n}(z)$ is the modified Bessel function of the second kind, and $\sum_{\vec{n} \neq 0} \equiv \sum_{n_{x}=-\infty}^{\infty} \sum_{n_{y}=-\infty}^{\infty} \sum_{n_{z}=-\infty}^{\infty}(1-$ $\delta(|\vec{n}|, 0))$ with $\vec{n}=\left(n_{x}, n_{y}, n_{z}\right)$.

Following the same procedure, one can obtain the FVCs of the other loop diagrams in Fig. 1. For the NNLO one-loop diagram of Fig. 1(c), one obtains

$$
\left.\delta G_{D}^{(c)}\left(M_{\phi}\right)=\frac{5}{36} \int_{0}^{1} d x m_{D}(x-2)\left[\delta_{1 / 2}\left(\mathcal{M}_{D}^{(c)}\right)^{2}\right)-\mathcal{M}_{D}^{(c)^{2}} \delta_{3 / 2}\left(\mathcal{M}_{D}^{(c)^{2}}\right)\right]
$$

with $\mathcal{M}_{D}^{(c)^{2}}=x^{2} m_{D}^{2}+(1-x) M_{\phi}^{2}-i \epsilon$. Taking the limit of $m_{D} \rightarrow \infty$, Eq. (15) and Eq. (17) reduce to

$$
\begin{aligned}
& \delta G_{D}^{(b)}\left(M_{\phi}\right)_{\mathrm{HB}}=-\frac{1}{8} \int_{0}^{\infty} d x\left[\delta_{1 / 2}\left(\beta_{\Delta}^{2}\right)-\beta_{\Delta}^{2} \delta_{3 / 2}\left(\beta_{\Delta}^{2}\right)\right], \\
& \delta G_{D}^{(c)}\left(M_{\phi}\right)_{\mathrm{HB}}=\frac{1}{2} \int_{0}^{\infty} d x\left[\delta_{1 / 2}\left(\beta^{2}\right)-\beta^{2} \delta_{3 / 2}\left(\beta^{2}\right)\right],
\end{aligned}
$$

where $\beta_{\Delta}=x^{2}-2 x \delta+M_{\phi}^{2}$ and $\beta=x^{2}+M_{\phi}^{2}$. They agree with the HB ChPT results of Ref. [59].

FVCs to the $\mathrm{N}^{3} \mathrm{LO}$ one-loop diagrams in Figs. 1(e), (f), and (g) have the following form:

$$
\begin{gathered}
\delta G_{D}^{(e, 1)}\left(M_{\phi}\right)=\frac{1}{2} \delta_{1 / 2}\left(M_{\phi}^{2}\right) \\
\delta G_{D}^{(e, 2)}\left(M_{\phi}\right)=\frac{1}{2} M_{\phi}^{2} \delta_{1 / 2}\left(M_{\phi}^{2}\right) \\
\delta G_{D}^{(e, 3)}\left(M_{\phi}\right)=\frac{1}{2} m_{D} \delta_{-1 / 2}\left(M_{\phi}^{2}\right) \\
\delta G_{D, B}^{(f)}\left(M_{\phi}\right)=\frac{1}{12} \int_{0}^{1} d x\left\{\left[m_{D}^{(2)}(x-1)-m_{B}^{(2)}\right] \cdot \delta_{1 / 2}\left(\mathcal{M}_{D}^{(b)^{2}}\right)\right. \\
+\left[(1-x)\left(2 \mathcal{M}_{D}^{(b)^{2}}+M_{\phi}^{2}(x-1)-\left(m_{0}+m_{D}\right)\left(m_{0}+2 m_{D}\right) x+2 m_{D}^{2} x^{2}\right) m_{D}^{(2)}\right. \\
\left.+\left(\mathcal{M}_{D}^{(b)^{2}}+3 m_{0} x\left(m_{0}+m_{D}(1-x)\right)\right) m_{B}^{(2)}\right] \cdot \delta_{3 / 2}\left(\mathcal{M}_{D}^{(b)^{2}}\right) \\
+ \\
\mathcal{M}_{D}^{(b)^{2}}\left[(x-1)\left(\mathcal{M}_{D}^{(b)}{ }^{2}+M_{\phi}^{2}(x-1)-\left(m_{0}+m_{D}\right)\left(m_{0}+2 m_{D}\right) x+2 m_{D}^{2} x^{2}\right) m_{D}^{(2)}\right. \\
\left.\left.\left.\quad-3 m_{0} x\left(m_{0}+m_{D}(1-x)\right) m_{B}^{(2)}\right] \cdot \delta_{5 / 2}\left(\mathcal{M}_{D}^{(b)}\right)^{2}\right)\right\}
\end{gathered}
$$




$$
\begin{aligned}
\delta G_{D, D^{\prime}}^{(g)}\left(M_{\phi}\right)= & \frac{5}{36} \int_{0}^{1} d x\left\{\left[(3 x-5) m_{D}^{(2)}+(3-2 x) m_{D^{\prime}}^{(2)}\right] \cdot \delta_{1 / 2}\left(\mathcal{M}_{D}^{(c)^{2}}\right)\right. \\
+ & {\left[\left(\mathcal{M}_{D}^{(c)^{2}}(10-6 x)+2 m_{D}^{2} x(2 x-3)+M_{\phi}^{2}\left(-3 x^{2}+8 x-5\right)\right) m_{D}^{(2)}\right.} \\
& \left.\left.+\left(\mathcal{M}_{D}^{(c)^{2}}(2 x-3)-3 m_{D}^{2}\left(x^{2}-2 x\right)\right) m_{D^{\prime}}^{(2)}\right] \cdot \delta_{3 / 2}\left(\mathcal{M}_{D}^{(c)}\right)^{2}\right) \\
+ & {\left[\mathcal{M}_{D}^{(c)^{2}}\left(m_{D}^{2}\left(6 x-4 x^{2}\right)+\mathcal{M}_{D}^{(c)}{ }^{2}(3 x-5)+M_{\phi}^{2}(x-1)(3 x-5)\right) m_{D}^{(2)}\right.} \\
& \left.\left.+3 \mathcal{M}_{D}^{(c)^{2}} m_{D}^{2}\left(x^{2}-2 x\right) m_{D^{\prime}}^{(2)}\right] \cdot \delta_{5 / 2}\left(\mathcal{M}_{D}^{(c)^{2}}\right)\right\} .
\end{aligned}
$$

The above standard procedure applies only to the case where $m_{D} \leq m_{0}+M_{\phi}$. For the case of $m_{D}>m_{0}+M_{\phi}$, we follow the approach proposed in Ref. [68] and replace the original $\delta_{r}\left(\mathcal{M}^{2}\right)$ with three parts by introducing a new scale $\mu$ satisfying $\mu<m_{0}+M_{\phi}$, i.e.,

$$
\delta_{r}\left(\mathcal{M}^{2}\right)=g_{1}^{r}-g_{2}^{r}+g_{3}^{r},
$$

where the $g_{1,2,3}^{r}$ are defined as

$$
\begin{aligned}
& g_{1}^{r}=\frac{1}{L^{3}} \sum_{\vec{k}}\left\{\frac{1}{\left[\frac{4 \pi^{2} \vec{n}^{2}}{L^{2}}+\mathcal{M}^{2}\left(m_{D}^{2}\right)\right]^{r}}-\frac{1}{\left[\frac{4 \pi^{2} \vec{n}^{2}}{L^{2}}+\mathcal{M}^{2}\left(\mu^{2}\right)\right]^{r}}+\frac{r\left(x^{2}-x\right)\left(m_{D}^{2}-\mu^{2}\right)}{\left[\frac{4 \pi^{2} \vec{n}^{2}}{L^{2}}+\mathcal{M}^{2}\left(\mu^{2}\right)\right]^{r+1}}\right\}, \\
& g_{2}^{r}=\int_{0}^{+\infty} \frac{k^{2} d k}{2 \pi^{2}} \cdot\left\{\frac{1}{\left[\vec{k}^{2}+\mathcal{M}^{2}\left(m_{D}^{2}\right)\right]^{r}}-\frac{1}{\left[\vec{k}^{2}+\mathcal{M}^{2}\left(\mu^{2}\right)\right]^{r}}+\frac{r\left(x^{2}-x\right)\left(m_{D}^{2}-\mu^{2}\right)}{\left[\vec{k}^{2}+\mathcal{M}^{2}\left(\mu^{2}\right)\right]^{r+1}}\right\}, \\
& g_{3}^{r}=\delta_{r}\left(\mathcal{M}^{2}\left(\mu^{2}\right)\right)-r\left(x^{2}-x\right)\left(m_{D}^{2}-\mu^{2}\right) \delta_{r+1}\left(\mathcal{M}^{2}\left(\mu^{2}\right)\right),
\end{aligned}
$$

with

$$
\begin{aligned}
\mathcal{M}^{2}\left(m_{D}^{2}\right) & =\left(x^{2}-x\right) m_{D}^{2}+x m_{0}^{2}+(1-x) M_{\phi}^{2}-i \epsilon \\
\mathcal{M}^{2}\left(\mu^{2}\right) & =\left(x^{2}-x\right) \mu^{2}+x m_{0}^{2}+(1-x) M_{\phi}^{2}-i \epsilon
\end{aligned}
$$

To take into account the FVCs in the study of the LQCD data, one simply replaces the loop functions $H$ of Eq. (13) by $\tilde{H}=H+\delta G$ with the $\delta G$ s calculated above.

\section{RESULTS AND DISCUSSION}

In this section, we perform a simultaneous fit of the $n_{f}=2+1$ LQCD data from the PACSCS [3], QCDSF-UKQCD [8], and HSC [6] Collaborations and the experimental data [11] to determine the 17 unknown LECs, $m_{D}, t_{D}, t_{0 \ldots 9}$, and $e_{1 \ldots 5}$. Since $t_{1}, t_{2}, t_{3}, t_{4}, t_{5}$, and $t_{6}$ appear in combinations, effectively we have only 14 independent LECs. The pion or light-quark mass dependence 
of the decuplet baryon masses is studied in the NLO, NNLO, and $\mathrm{N}^{3} \mathrm{LO}$ EOMS BChPT. Using the so-obtained LECs, we also carry out a detailed study on the QCDSF-UKQCD and LHPC data to test the applicability of the $\mathrm{N}^{3} \mathrm{LO}$ BChPT and the consistency between different LQCD simulations. Furthermore, the pion- and strangeness-baryon sigma terms are predicted by the use of the Feynman-Hellmann theorem.

\section{A. LQCD data and values of LECs}

Up to now, five collaborations have reported $n_{f}=2+1$ simulations of the decuplet baryon masses, i.e., the BMW [1], PACS-CS [3], LHPC [5], HSC [6], and QCDSF-UKQCD [8] Collaborations. Because the BMW data are not publicly available and the data of the LHPC Collaboration seem to suffer some systematic errors, as shown in their chiral extrapolation result on the $\Delta(1232)$ mass, which is much higher than its physical value [5] (see also Sec. [II B), we will concentrate on the data of the PACS-CS, QCDSF-UKQCD, and HSC Collaborations. Following the criteria used in our previous studies [58], we only select the LQCD data that satisfy $M_{\pi}<0.5 \mathrm{GeV}$ and $M_{\phi} L>3.8$. As a result, there are eight sets of data from the PACS-CS (3 sets), QCDSF-UKQCD (2 sets), and HSC (3 sets) Collaborations. Among the eight LQCD data sets studied, only in the ensemble with $M_{\pi}=296 \mathrm{MeV}$ from the PACS-CS Collaboration, can the decay $\Delta \rightarrow N+\pi$ happen. It should be noted that the PACS-CS Collaboration measured the lowest energy levels of the vector meson and decuplet baryon channels, which are different from the true resonance masses. The resulting difference for the $\rho$ meson is estimated to be 5 percent using Lüscher's formula [3]. We will comment on this later.

It should be mentioned that the $O(a)$-improved Wilson action was used by all the above collaborations except the LHPC Collaboration, which employed a mixed action. The $O(a)$-improved action has the favorable property that the leading order corrections from the finite lattice spacing are eliminated. The finite lattice spacing corrections of the mixed action of the LHPC Collaboration were also shown to be small [5]. Therefore, in the present work we assume that the discretization artifacts of the present LQCD simulations are small and can be ignored, and will leave a detailed study on finite lattice spacing artifacts to a future study (for a recent study of the discretization effects on the octet baryon masses, see Ref. [69]).

Before we perform a simultaneous fit of the LQCD data, we specify our strategy to fix some

of the LECs in the $\mathrm{N}^{3} \mathrm{LO}$ BChPT mass formulas [Eq. (13)]. For the meson-decay constant, we 
use $F_{\phi}=0.0871 \mathrm{GeV}$. The $\phi B D$ coupling is fixed to the $\mathrm{SU}(3)$-average value among the different decuplet-to-octet pionic decay channels, $C=0.85$ [70]. The $\phi D D$ coupling $\mathcal{H}$ is barely known, and we fix it using the large $N_{c}$ relation $H_{A}=(9 / 5) g_{A}$, where $g_{A}$ and $H_{A}$ are the nucleon and $\Delta$ axial charges. With $g_{A}=1.26$, this yields the $\phi D D$ coupling $\mathcal{H}=H_{A} / 2=1.13$. In the loop function Eq. (24), the LO corrections to the virtual octet masses are included; therefore, there are four more LECs $m_{0}, b_{0}, b_{D}$, and $b_{F}$ related to the octet baryon masses up to $O\left(p^{2}\right)$. Similar to the determination of the decuplet baryon masses at $O\left(p^{2}\right)$ [58], their values can be obtained by fitting the physical octet baryon masses with the NLO octet mass formula $M_{B}=m_{0}-m_{B}^{(2)}$. Because at the same pion masses, the $m_{0}$ and $b_{0}$ cannot be disentangled, we only obtain $m_{0}^{\text {eff }}=m_{0}-b_{0}\left(4 M_{K}^{2}+2 M_{\pi}^{2}\right)$, $b_{D}=0.06 \mathrm{GeV}^{-1}$, and $b_{F}=-0.231 \mathrm{GeV}^{-1}$. The octet-decuplet mass splitting $\delta=0.231 \mathrm{GeV}$ is taken as the average gap of the physical octet and decuplet masses. As a result, $m_{0}$ and $b_{0}$ can be expressed as $m_{0}=m_{D}-0.231 \mathrm{GeV}$ and $b_{0}=\left(m_{D}-1.423\right) / 1.014 \mathrm{GeV}^{-1}$.

In the fitting process, we incorporate the inverse of the correlation matrix $C_{i j}=\sigma_{i} \sigma_{j} \delta_{i j}+\Delta a_{i} \Delta a_{j}$ for each lattice ensemble to calculate the $\chi^{2}$, where $\sigma_{i}$ are the lattice statistical errors and the $\Delta a_{i}$ are the fully correlated errors propagated from the determination of $a_{i}$. This is because the data from different collaborations are not correlated with each other, but the data from the same collaboration are partially correlated by the uncertainties propagated from the determination of the lattice spacing $a$.

\section{B. Light-quark mass dependence of the decuplet baryon masses}

In this subsection, we proceed to study the eight sets of LQCD data for the decuplet baryon masses by using the $\mathrm{N}^{3} \mathrm{LO}$ BChPT mass formulas [Eq. (13)]. In order to constrain better the values of the LECs, we include the precise experimental data in the fitting. The obtained 14 LECs from the best fits are tabulated in Table III. For the sake of comparison, we also perform fits at NLO 4 and NNLO. Up to NNLO, there are only three LECs, i.e., $m_{D}, t_{0}$, and $t_{D}$.

It is clear that the NLO fit (without loop contributions) already describes the LQCD simulations very well. The description becomes a bit worse at NNLO 5 While the description at $\mathrm{N}^{3} \mathrm{LO}$ becomes much better, yielding a $\chi^{2} /$ d.o.f. $=0.20$. Therefore we confirm that the PACS-CS,

${ }^{4}$ Because at $O\left(p^{2}\right)$ BChPT does not generate any FVCs, we have adjusted the lattice data by subtracting the FVCs calculated by the $\mathrm{N}^{3}$ LO EOMS BChPT.

${ }^{5}$ Without the contributions of the virtual octet baryons, the NNLO description would be much better, with a $\chi^{2} /$ d.o.f. $\approx 2.9$. 
TABLE III. Values of the LECs from the best fits to the LQCD data and the experimental data with different fitting strategies at $O\left(p^{2}\right), O\left(p^{3}\right)$, and $O\left(p^{4}\right)$, respectively. The estimator for the fits with and without the experimental decuplet masses, $\chi^{2} /$ d.o.f. and $\chi^{2} /$ d.o.f. ${ }^{*}$, are given in the last two rows (see text for details).

\begin{tabular}{lccc}
\hline \hline & $\mathrm{NLO}$ & $\mathrm{NNLO}$ & $\mathrm{N}^{3} \mathrm{LO}$ \\
\hline$m_{D}[\mathrm{MeV}]$ & $1135(14)$ & $870(12)$ & $1152(25)$ \\
$t_{0}\left[\mathrm{GeV}^{-1}\right]$ & $0.167(27)$ & $1.36(2)$ & $0.0710(59)$ \\
$t_{D}\left[\mathrm{GeV}^{-1}\right]$ & $0.322(2)$ & $0.785(3)$ & $0.318(16)$ \\
$\tilde{t}_{1}\left[\mathrm{GeV}^{-1}\right]$ & - & - & $5.90(24)$ \\
$\tilde{t}_{2}\left[\mathrm{GeV}^{-1}\right]$ & - & - & $-2.26(29)$ \\
$\tilde{t}_{3}\left[\mathrm{GeV}^{-1}\right]$ & - & - & $-3.67(45)$ \\
$t_{7}\left[\mathrm{GeV}^{-2}\right]$ & - & - & $-2.37(8)$ \\
$t_{8}\left[\mathrm{GeV}^{-2}\right]$ & - & - & $0.298(156)$ \\
$t_{9}\left[\mathrm{GeV}^{-2}\right]$ & - & - & $1.21(13)$ \\
$e_{1}\left[\mathrm{GeV}^{-3}\right]$ & - & - & $-0.00386(11689)$ \\
$e_{2}\left[\mathrm{GeV}^{-3}\right]$ & - & - & $0.194(47)$ \\
$e_{3}\left[\mathrm{GeV}^{-3}\right]$ & - & - & $-0.167(117)$ \\
$e_{4}\left[\mathrm{GeV}^{-3}\right]$ & - & - & $0.0767(480)$ \\
$e_{5}\left[\mathrm{GeV}^{-3}\right]$ & - & - & $-0.0182(734)$ \\
\hline$\chi^{2} / \mathrm{d} . o . f .^{2} / \mathrm{d} . o . f .^{*}$ & 4.4 & 9.5 & 0.20 \\
\hline \hline
\end{tabular}

QCDSF-UKQCD, and HSC data are consistent with each other, although their setups are different. Furthermore, it seems that the LQCD decuplet baryon masses are almost linear in $M_{\pi}^{2}$, as demonstrated by the good fit obtained at NLO, $\chi^{2} /$ d.o.f. $^{*}=0.44$.

The values of the 14 LECs seem very natural, except that the LECs $\tilde{t}_{1}, \tilde{t}_{2}, \tilde{t}_{3}$, and $t_{7}$ might be slightly large. If we had constrained their values to lie between -1 to 1 in the fitting process, we would have obtained a $\chi^{2} /$ d.o.f. $=0.23$, instead of 0.20 . It is evident that the present LQCD 
simulations are not precise enough or are too limited to put a stringent constraint on the values of all the LECs appearing up to $\mathrm{N}^{3} \mathrm{LO}$, because the NLO fit already yields a $\chi^{2} /$ d.o.f. ${ }^{*}$ smaller than 1 . This is further confirmed by the relatively large correlation observed between some of the LECs, e.g., between $\tilde{t}_{1}$ and $\tilde{t}_{2}$, among $t_{7}, t_{8}$, and $t_{9}$, and among $e_{1}, e_{3}$, and $e_{5}$. We found that putting some of these LECs to zero only slightly increases the $\chi^{2} /$ d.o.f.. In short, the values of the $\mathrm{N}^{3}$ LO LECs and the corresponding uncertainties should be viewed in the present context and used with care.

As mentioned earlier, the lightest LQCD point with $M_{\pi}=296 \mathrm{MeV}$ of the PACS-CS Collaboration suffers from potentially large systematic errors. If we had performed the fit without this point, we would have obtained a $\chi^{2} /$ d.o.f. $=0.24$, slightly larger than the $\chi^{2} /$ d.o.f. $=0.20$ of Table In addition, the values of the corresponding LECs would change moderately. On the other hand, the extrapolations with the LECs determined from the fit excluding the physical masses became much worse. This seems to suggest that the inclusion of the lightest PACS-CS point is reasonable, keeping in mind the caveat that they may suffer from potentially large systematic errors. This is also the strategy adopted by the PACS-CS Collaboration [59] and other similar studies [54].

In Fig. 2, we show the $\Delta, \Sigma^{*}, \Xi^{*}$, and $\Omega^{-}$masses as functions of $M_{\pi}^{2}$, where the strange-quark mass is set at its physical value. It is clear that the LQCD data are rather linear in $M_{\pi}^{2}$. The $O\left(p^{3}\right)$ BChPT results show strong curvature and cannot describe the LQCD data. A good description can only be achieved up to $\mathrm{N}^{3} \mathrm{LO} 6$ In Fig. 2, we also show those data of the PACS-CS and HSC Collaborations that are excluded from the fit. The $O\left(p^{4}\right)$ BChPT can describe reasonably well those data as well.

It should be emphasized that the setups of the QCDSF-UKQCD simulations are rather different from those of the PACS-CS and HSC Collaborations. Most LQCD simulations fix the strangequark mass at (or close to ) its physical value and gradually moving the $u / d$ quark masses to their physical values. The QCDSF-UKQCD Collaboration adopted an alternative method by starting at a point on the $\mathrm{SU}(3)$ flavor symmetric line $\left(m_{u / d}=m_{s}\right)$ and holding the sum of the quark masses $\bar{m}=\left(2 m_{u / d}+m_{s}\right) / 3$ constant [7]. In this way, the corresponding kaon and eta masses can be smaller than the pion mass. On the other hand, the FVCs from the kaon and eta loops can become comparable or even larger than that induced by the pion loop, because the $M_{\phi} L$ can simultaneously become smaller than 4. Therefore, the QCDSF-UKQCD data provide us an opportunity to test the BChPT in the world of small strange-quark masses and small lattice volumes.

\footnotetext{
${ }^{6}$ In principle, at NNLO, we can use for the meson-decay constant its $\mathrm{SU}(3)$ average, $F_{\phi}=1.17 f_{\pi}$ with $f_{\pi}=92.4$ MeV. This improves a lot the NNLO fit.
} 


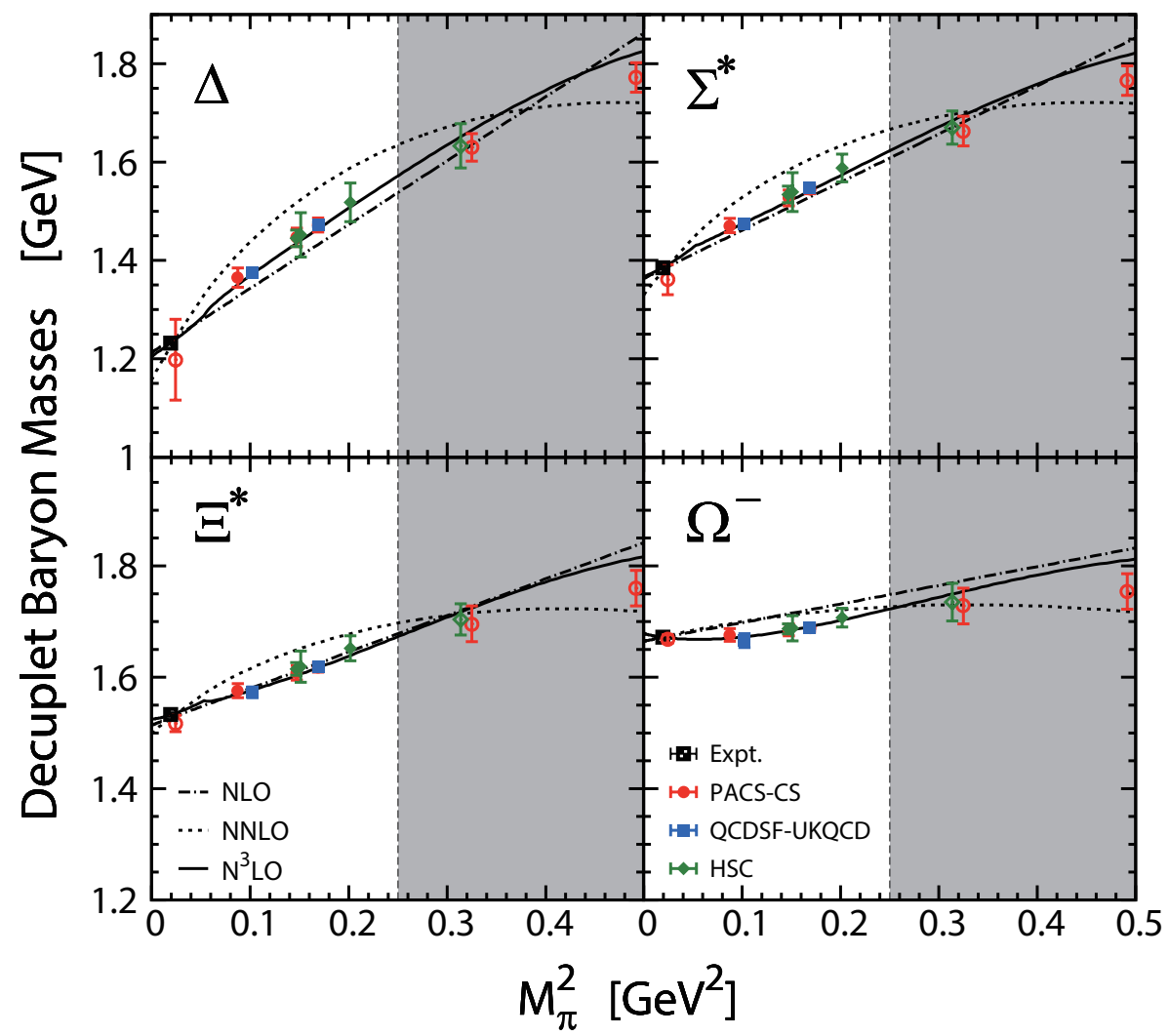

FIG. 2. (Color online). Pion mass dependence of the lowest-lying decuplet baryon masses. Filled (open) symbols denote the lattice data points included in (excluded from) the fits, which are projected to have the physical strange-quark mass. The dot-dashed, the dashed, and the solid lines are the best NLO, NNLO and $\mathrm{N}^{3} \mathrm{LO}$ fits to the lattice data, respectively. In obtaining the BChPT results, the strange-quark mass has been set to its physical value. The lattice points in the shaded region are not included in the fits.

In Fig. 3, the QCDSF-UKQCD data are compared with the $\mathrm{N}^{3} \mathrm{LO}$ BChPT. The LQCD points included in the fit are denoted by solid points and those excluded from the fit by hollow points. All lattice points are shifted by FVCs and the kaon mass is fixed using the function $M_{K}^{2}=a+b M_{\pi}^{2}$ for the lattice ensemble with $a$ and $b$ determined in Appendix II of Ref. [57]. It is clear that the $\mathrm{N}^{3} \mathrm{LO}$ BChPT can describe reasonably well the QCDSF-UKQCD data obtained in both large $\left(N_{s}=32\right)$ and small $\left(N_{s}=24\right)$ volumes with both heavy and light pion masses. However, it should be pointed out that the ratio method eliminates to a large extent the FVCs. In other words, to plot/study the data this way one can neglect FVCs, as noticed in Ref. [8].

In Table IV, we show the FVCs to the LQCD data calculated in the $\mathrm{N}^{3}$ LO BChPT. Most of them are at the order of a few of tens of MeV. Among them, the FVCs to the QCDSF-UKQCD 


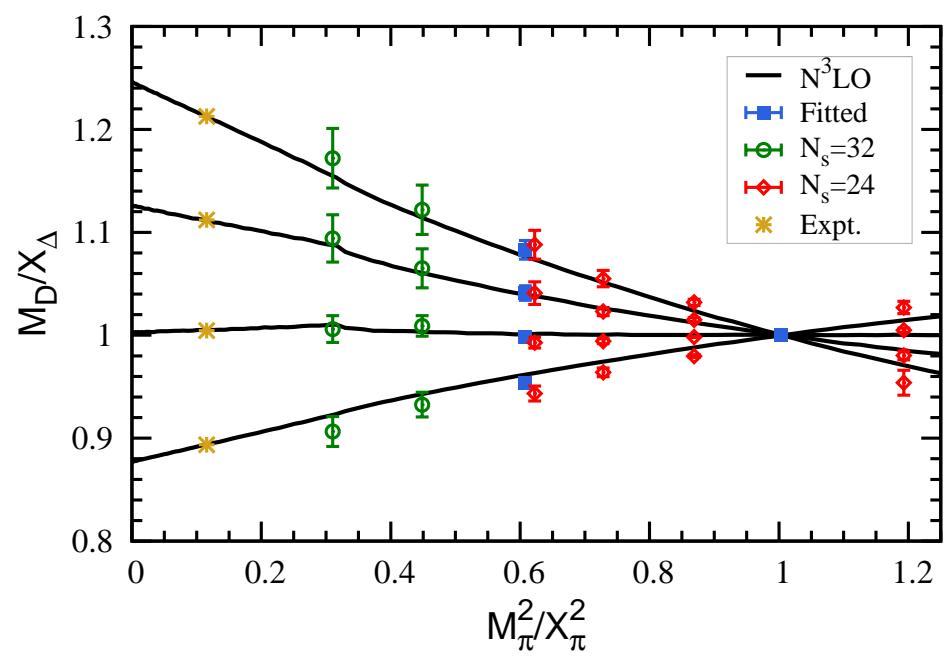

FIG. 3. (Color online). The QCDSF-UKQCD lattice data [7] in comparison with the $\mathrm{N}^{3} \mathrm{LO}$ BChPT. The lattice data denoted by the blue filled squares are included in the fit; those by the green opened circles (with $N_{s}=32$ ) and the red diamonds (with $N_{s}=24$ ) are not. FVCs of the lattice results have been subtracted. The two-flavour singlet quantities, $X_{\pi}$ and $X_{\Delta}$, are defined as, $X_{\pi}=\sqrt{\left(M_{\pi}^{2}+2 M_{K}^{2}\right) / 3}, X_{\Delta}=\left(2 m_{\Delta}+m_{\Omega^{-}}\right) / 3$, respectively [7].

data are the largest, which can be easily understood from the arguments given above.

TABLE IV. Finite-volume corrections (in units of MeV) to LQCD decuplet baryon masses in covariant BChPT up to $\mathrm{N}^{3} \mathrm{LO}$.

\begin{tabular}{c|cc|cccc|ccc}
\hline \hline & $M_{\pi}$ & $M_{K}$ & $\delta m_{\Delta}$ & $\delta m_{\Sigma^{*}}$ & $\delta m_{\Xi^{*}}$ & $\delta m_{\Omega^{-}}$ & $M_{\pi} L$ & $M_{K} L$ & $M_{\eta} L$ \\
\hline \multirow{2}{*}{ PACS-CS } & 296 & 594 & 14 & 5 & 0 & -3 & 4.3 & 8.7 & 9.8 \\
& 384 & 581 & 5 & 2 & 1 & 1 & 5.7 & 8.6 & 9.3 \\
& 411 & 635 & 4 & 2 & 0 & 1 & 6.0 & 9.3 & 10.2 \\
\hline \multirow{2}{*}{ QCDSF-UKQCD } & 320 & 451 & 20 & 13 & 8 & 4 & 4.1 & 5.8 & 6.2 \\
& 411 & 411 & 50 & 50 & 50 & 50 & 3.95 & 3.95 & 3.95 \\
\hline \multirow{2}{*}{ HSC } & 383 & 544 & 4 & 2 & 1 & 0 & 5.7 & 8.1 & 8.8 \\
& 389 & 546 & 42 & 27 & 14 & 3 & 3.9 & 5.4 & 5.9 \\
& 449 & 581 & 28 & 19 & 11 & 4 & 4.5 & 5.8 & 6.2 \\
\hline \hline
\end{tabular}




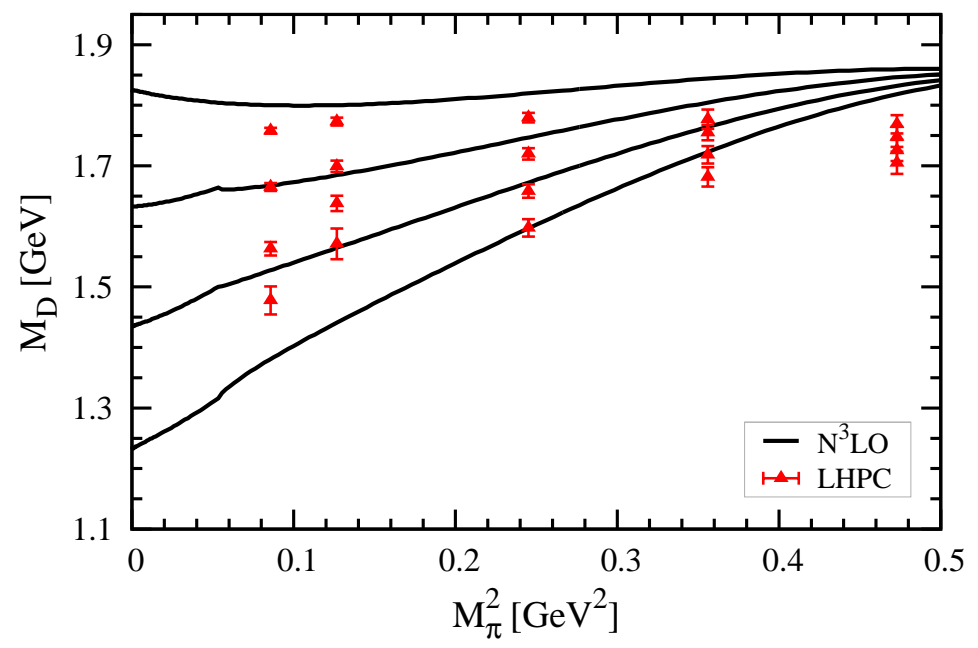

FIG. 4. (Color online). Comparison between the $\mathrm{N}^{3}$ LO BChPT and the LHPC data [5].

We would like to point out that in the above fits we have not included the LHPC data, while in Refs. [57, 58] we have studied their data for the octet baryon masses. The reason is that the LHPC decuplet baryon data do not seem to be consistent with those of the PACS-CS, QCDSF-UKQCD, and HSC Collaborations. This is clearly demonstrated in Fig. 4, where the LHPC data are contrasted with the $\mathrm{N}^{3}$ LO BChPT with the $\mathrm{N}^{3}$ LO LECs tabulated in Table $\Pi$, and the corresponding kaon mass is fixed using $M_{K}^{2}=a+b M_{\pi}^{2}$ with $a$ and $b$ determined in Ref. [57]. It is clear that the dependencies of the lattice data on $M_{\pi}^{2}$ seem to be flatter than suggested by the $\mathrm{N}^{3} \mathrm{LO}$ BChPT. In Ref. [5], it was noticed that it is difficult to extrapolate the LQCD data to the physical $\Delta(1232)$ mass. Our study seems to confirm their finding. If we had included the LHPC data ${ }^{7}$ (three sets of them satisfying our selection criteria) in our fitting, we would have obtained a $\chi^{2} /$ d.o.f. $=2.2$.

Furthermore, in order to quantify the effects of loop contributions involving virtual octet and decuplet baryons, one can allow $\mathcal{C}$ and $\mathcal{H}$ to vary in the fitting. The corresponding $\chi^{2} /$ d.o.f. from the best fit is 0.23 with $C=0.75$ and $\mathcal{H}=1.0$. It is clear that the values are consistent with the phenomenological values we used above, which can be seen as evidence for the existence of non-analytical chiral contributions following the argument given in Ref. [71]. One should note, however, that because of the small difference between the $\chi^{2} /$ d.o.f. obtained here and the $\chi^{2} /$ d.o.f. obtained by putting $C$ and $\mathcal{H}$ to zero, this evidence is rather weak in the present case.

\footnotetext{
${ }^{7}$ It needs to be mentioned that in Ref. [71], a different way of setting the lattice scale has been used to obtain the decuplet baryon masses of the LHPC Collaboration [5] in physical units.
} 


\section{Convergence of SU(3) EOMS BChPT}

Convergence of BChPT in the $u, d$, and $s$ three-flavor sector has been under debate for many years. See, e.g., Refs.[28, 66, 72, 73] and references cited therein 8 One prominent example is the magnetic moments of octet baryons. In Ref. [76], it has been shown that compared to the HB ChPT and the IR BChPT, the EOMS BChPT converges relatively faster. The same has been found for the octet baryon masses [50]. Nevertheless, even in the EOMS BChPT, convergence is relatively slow because of the large expansion parameter, $M_{K} / \Lambda_{\mathrm{ChPT}}$. Naively, each higher-order contribution is only suppressed by about one-half at the physical point, which can even be further reduced for LQCD simulations with larger light-quark masses. To speed up convergence, several alternative formulations of BChPT have been proposed, such as the long distance regularization method [72], the cutoff scheme [28], and finite-range regulator method [27, 73] BChPT, which exhibit better convergence by suppressing loop contributions with either a cutoff or a form factor.

In the following, we would like to examine the contributions of different chiral orders. In Table 【II and Fig. 2, one notices that the NLO BChPT can already describe the LQCD data very well, but the experimental data are missed a little bit. Naturally one would expect that up to NNLO and $\mathrm{N}^{3} \mathrm{LO}$, there should be some reshuffling of contributions of different orders. This can be clearly seen from Table $\mathrm{V}$, where contributions of different chiral orders to the decuplet baryon masses at the physical point are tabulated. On the other hand, once loop diagrams are included, a naive comparison of $p^{0}\left(m_{D}\right), p^{2}, p^{3}$, and $p^{4}$ contributions turns out to be troubling. At NNLO, the

TABLE V. Contributions of different chiral orders to the decuplet baryon masses at the physical point (in units of $\mathrm{GeV}$ ).

\begin{tabular}{|c|c|c|c|c|c|c|c|c|c|c|c|c|c|}
\hline & \multirow[b]{2}{*}{$m_{D}$} & \multicolumn{3}{|c|}{$\Delta$} & \multicolumn{3}{|c|}{$\Sigma^{*}$} & \multicolumn{3}{|c|}{$\Xi^{*}$} & \multicolumn{3}{|c|}{$\Omega^{-}$} \\
\hline & & $p^{2}$ & $p^{3}$ & $p^{4}$ & $p^{2}$ & $p^{3}$ & $p^{4}$ & $p^{2}$ & $p^{3}$ & $p^{4}$ & $p^{2}$ & $p^{3}$ & $p^{4}$ \\
\hline NLO & 1.135 & 0.104 & - & - & 0.248 & - & - & 0.392 & - & - & 0.537 & - & - \\
\hline NNLO & 0.870 & 0.737 & -0.383 & - & 1.089 & -0.582 & - & 1.441 & -0.785 & - & 1.793 & -0.991 & - \\
\hline $\mathrm{N}^{3} \mathrm{LO}$ & 1.152 & 0.046 & -0.429 & 0.463 & 0.158 & -0.652 & 0.728 & 0.270 & -0.878 & 0.988 & 0.382 & -1.106 & 1.244 \\
\hline
\end{tabular}

\footnotetext{
${ }^{8}$ For related discussions in the mesonic sector, see, e.g., Refs. [74, 75], where the so-called resummed chiral perturbation theory has been shown to exhibit better convergence than conventional chiral perturbation theory. To our knowledge, no similar studies exist in the one-baryon sector.
} 


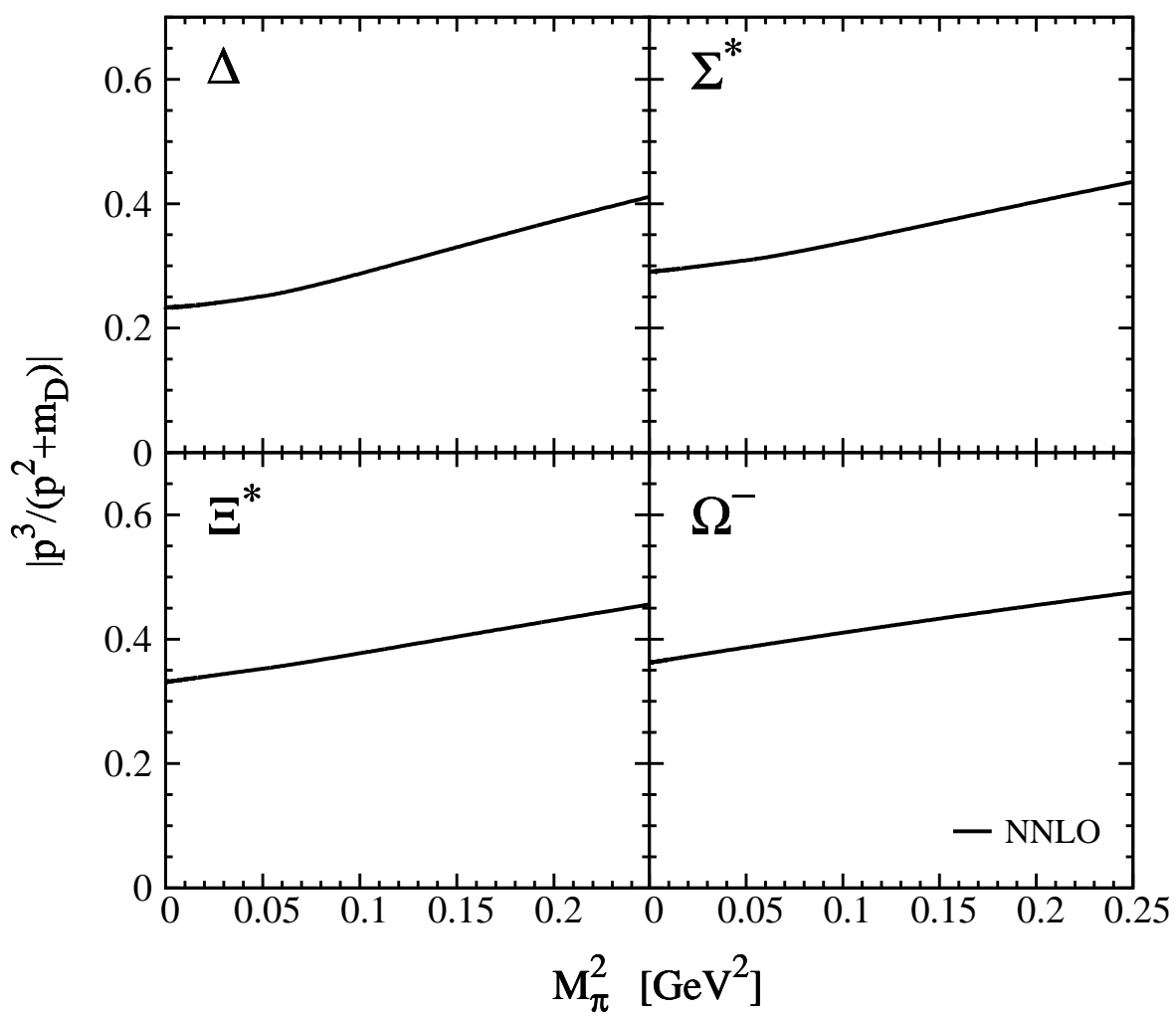

FIG. 5. Ratio of one-loop and tree contributions to the decuplet baryon masses, $\left|p^{3} /\left(p^{2}+m_{D}\right)\right|$, as a function of pion mass. The strange-quark mass is set at its physical value.

$p^{2}$ contributions can be a factor of 2 larger than $m_{D}$, while at $\mathrm{N}^{3} \mathrm{LO}$, the $p^{3}$ and $p^{4}$ contributions are opposite and become comparable to or even larger than the $p^{2}$ contributions, particularly for the decuplet baryons containing strangeness.

On the other hand, up to one-loop level, it might be more proper to judge convergence by comparing tree-level and loop contributions. In Figs. 5 and $6,\left|p^{3} /\left(p^{2}+m_{D}\right)\right|$ and $\left|\left(p^{3}+p^{4}\right) /\left(p^{2}+m_{D}\right)\right|$ are shown as a function of $M_{\pi}^{2}$. At NNLO, the $p^{3}$ contributions can reach about $50 \%$ of the treelevel contributions, while at $\mathrm{N}^{3} \mathrm{LO}$ the loop contributions become about $10 \% \sim 20 \%$ of the treelevel contributions. These results suggest that the chiral expansions are convergent as expected.

\section{Pion- and strangeness-baryon sigma terms}

The baryon sigma terms are important quantities in understanding the chiral condensate and the composition of the baryons. At present, there is no direct LQCD simulation of these quantities for the decuplet baryons. On the other hand, one can calculate the decuplet baryon sigma terms 


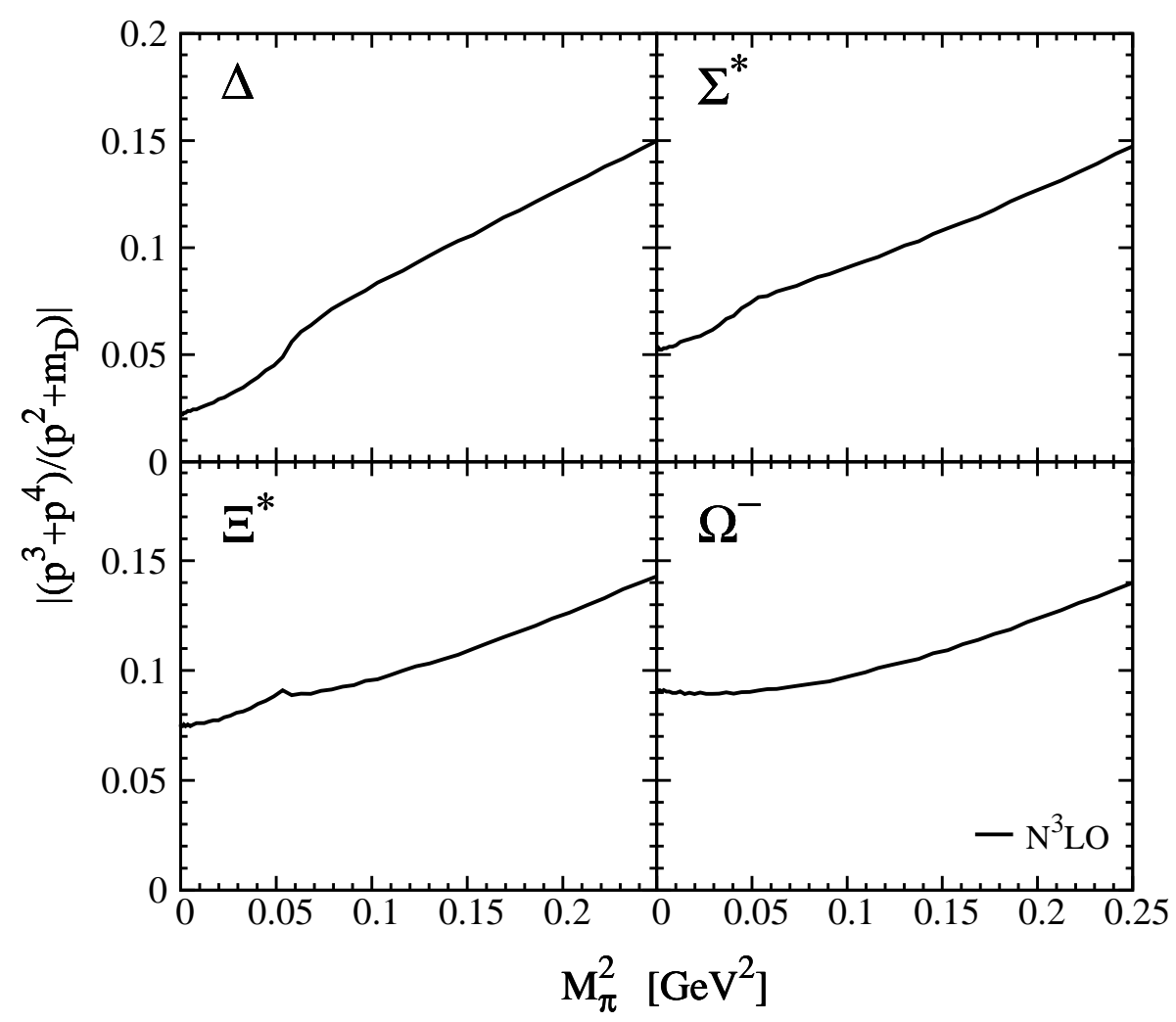

FIG. 6. Ratio of one-loop and tree contributions to the decuplet baryon masses, $\left|\left(p^{3}+p^{4}\right) /\left(p^{2}+m_{D}\right)\right|$, as a function of pion mass. The strange-quark mass is set at its physical value.

$\sigma_{\pi D}$ and $\sigma_{s D}$ using BChPT, once the relevant LECs are fixed, via the Feynman-Hellmann theorem by treating the decuplet baryons as stable particles as in standard BChPT. See, e.g., Ref. [57] for relevant formulas.

Using the LECs given in Table III, we calculate the sigma terms of the baryon decuplet at the physical point, and the results are listed in Table VI] For comparison, we also tabulate the results of Refs. [50, 54]. The difference between our $O\left(p^{3}\right)$ predictions with those of Ref. [50] reflects the influence of the LQCD data and the fitting strategy. While our $\mathrm{N}^{3} \mathrm{LO}$ results are consistent with those of Ref. [54] within uncertainties.

\section{SUMMARY}

We have studied the ground-state decuplet baryon masses in baryon chiral perturbation theory with the extended-on-mass-shell scheme up to next-to-next-to-next-to-leading order. Through a simultaneous fit of the $n_{f}=2+1$ LQCD data from the PACS-CS, QCDSF-UKQCD, and HSC 
TABLE VI. Pion- and strangeness-sigma terms of the decuplet baryons at the physical point. The first error is statistical and the second is systematic, estimated by taking half the difference between the $\mathrm{N}^{3} \mathrm{LO}$ result and the NNLO result.

\begin{tabular}{c|cc|cc}
\hline \hline & \multicolumn{2}{|c|}{ NNLO } & \multicolumn{2}{|c}{$\mathrm{N}^{3} \mathrm{LO}$} \\
\cline { 2 - 5 } & This work & Ref. [50] & This work & Ref. [54] \\
\hline$\sigma_{\pi \Delta}[\mathrm{MeV}]$ & $64(1)$ & $55(4)(18)$ & $28(1)(18)$ & $34(3)$ \\
$\sigma_{\pi \Sigma^{*}}[\mathrm{MeV}]$ & $44(1)$ & $39(3)(13)$ & $22(2)(11)$ & $28(2)$ \\
$\sigma_{\pi \Xi^{*}}[\mathrm{MeV}]$ & $26(1)$ & $22(3)(7)$ & $11(2)(8)$ & $18(4)$ \\
$\sigma_{\pi \Omega^{-}}[\mathrm{MeV}]$ & $8(1)$ & $5(2)(1)$ & $-5(2)(6)$ & $10(4)$ \\
\hline$\sigma_{s \Delta}[\mathrm{MeV}]$ & $93(12)$ & $56(24)(1)$ & $88(22)(3)$ & $41(41)$ \\
$\sigma_{s \Sigma^{*}}[\mathrm{MeV}]$ & $181(13)$ & $160(28)(7)$ & $243(24)(31)$ & $211(44)$ \\
$\sigma_{s \Xi^{*}}[\mathrm{MeV}]$ & $258(14)$ & $274(32)(9)$ & $391(24)(67)$ & $373(53)$ \\
$\sigma_{s \Omega^{-}}[\mathrm{MeV}]$ & $326(15)$ & $360(34)(26)$ & $528(26)(101)$ & $510(50)$ \\
\hline \hline
\end{tabular}

Collaborations, the 14 unknown low-energy constants are determined. In fitting the LQCD data, finite-volume corrections are taken into account self-consistently. A $\chi^{2} /$ d.o.f. $=0.20$ is achieved for the eight sets of LQCD data satisfying $M_{\pi}^{2}<0.25 \mathrm{GeV}^{2}$ and $M_{\phi} L>3.8$.

Our studies show that the chiral expansions are convergent as expected and the results of the PACS-CS, QCDSF-UKQCD, and HSC Collaborations seem to be consistent with each other, but not those of the LHPC Collaboration. We have calculated the sigma terms of the decuplet baryons by use of the Feynman-Hellmann theorem, which should be compared to future LQCD data.

It should be noted that our present study suffers from the limited range of the LQCD data (in terms of the input parameters) and the rather large number of unknown low-energy constants. Future refined LQCD simulations with various light-quark and strange-quark masses, lattice volume and lattice spacing will be extremely welcome to put covariant baryon chiral perturbation theory to a more stringent test than was done in the present work. In the context of effective field theories, one would like to apply the same formalism and utilize the same low-energy constants to study other related physical observables, which can also serve as an additional test. Such works are in progress and will be reported elsewhere. 


\section{ACKNOWLEDGMENTS}

X.-L.R thanks Dr. Hua-Xing Chen for useful discussions and acknowledges support from the Innovation Foundation of Beihang University for Ph.D. Graduates. L.-S.G acknowledges support from the Alexander von Humboldt foundation. This work was partly supported by the National Natural Science Foundation of China under Grants No. 11005007, No. 11035007, and No. 11175002, the New Century Excellent Talents in the University Program of Ministry of Education of China under Grant No. NCET-10-0029, the Fundamental Research Funds for the Central Universities, and the Research Fund for the Doctoral Program of Higher Education under Grant No. 20110001110087.

\section{APPENDIX}

Here we show explicitly the $\mathrm{N}^{3} \mathrm{LO}$ loop functions appearing in Eq. (13), which are calculated in the EOMS scheme:

$$
\begin{aligned}
& H_{D}^{(e, 1)}\left(M_{\phi}\right)=M_{\phi}^{2}\left[1+\ln \left(\frac{\mu^{2}}{M_{\phi}^{2}}\right)\right], \\
& H_{D}^{(e, 2)}\left(M_{\phi}\right)=M_{\phi}^{4}\left[1+\ln \left(\frac{\mu^{2}}{M_{\phi}^{2}}\right)\right], \\
& H_{D}^{(e, 3)}\left(M_{\phi}\right)=m_{D}\left\{\frac{M_{\phi}^{4}}{4}\left[1+\ln \left(\frac{\mu^{2}}{M_{\phi}^{2}}\right)\right]+\frac{1}{8} M_{\phi}^{4}\right\} .
\end{aligned}
$$




$$
\begin{aligned}
& H_{D, B}^{(f)}\left(M_{\phi}\right)=\frac{1}{144 m_{D}^{4}} m_{D}^{(2)} M_{\phi}^{2}\left[90 m_{0}^{4}+96 m_{0}^{3} m_{D}+36 m_{0}^{2} m_{D}^{2}+48 m_{0} m_{D}^{3}-22 m_{D}^{4}\right. \\
& \left.-3\left(30 m_{0}^{2}+16 m_{0} m_{D}+19 m_{D}^{2}\right) M_{\phi}^{2}+30 M_{\phi}^{4}\right] \\
& -\frac{1}{12 m_{D}^{3}} m_{B}^{(2)} M_{\phi}^{2}\left[8 m_{0}^{3}+8 m_{0}^{2} m_{D}+4 m_{0} m_{D}^{2}+7 m_{D}^{3}-\left(4 m_{0}+m_{D}\right) M_{\phi}^{2}\right] \\
& +\frac{1}{24 m_{D}^{6}} m_{D}^{(2)} M_{\phi}^{2} \ln \left(\frac{M_{\phi}}{m_{0}}\right)\left[4 m_{0}^{5}\left(5 m_{0}+6 m_{D}\right)\right. \\
& \left.-6\left(5 m_{0}^{4}+4 m_{0}^{3} m_{D}+3 m_{0}^{2} m_{D}^{2}+2 m_{0} m_{D}^{3}+m_{D}^{4}\right) M_{\phi}^{2}+4\left(5 m_{0}^{2}+2 m_{0} m_{D}+3 m_{D}^{2}\right) M_{\phi}^{4}-5 M_{\phi}^{6}\right] \\
& -\frac{1}{12 m_{D}^{5}} m_{B}^{(2)} M_{\phi}^{2} \ln \left(\frac{M_{\phi}}{m_{0}}\right)\left[3 m_{0}^{4}\left(4 m_{0}+5 m_{D}\right)\right. \\
& \left.-3\left(4 m_{0}^{3}+3 m_{0}^{2} m_{D}+2 m_{0} m_{D}^{2}+m_{D}^{3}\right) M_{\phi}^{2}+\left(4 m_{0}+m_{D}\right) M_{\phi}^{4}\right] \\
& +\frac{1}{24 m_{D}^{6}}\left(m_{0}-m_{D}\right)^{2}\left(m_{0}+m_{D}\right)^{4}\left[\ln \left(m_{0} M_{\phi}\right)-\ln \left(m_{0}^{2}-m_{D}^{2}\right)-i \pi\right] \\
& \times\left[2 m_{D} m_{B}^{(2)}\left(4 m_{0}-m_{D}\right)-m_{D}^{(2)}\left(5 m_{0}^{2}-2 m_{0} m_{D}+3 m_{D}^{2}\right)\right] \\
& +\frac{1}{12} M_{\phi}^{2}\left(3 m_{B}^{(2)}+2 m_{D}^{(2)}\right) \ln \left(\frac{m_{0} M_{\phi}}{\mu^{2}}\right) \\
& +\frac{1}{24 m_{D}^{6} \sqrt{\mathcal{W}}}\left(m_{0}^{2}-2 m_{0} m_{D}+m_{D}^{2}-M_{\phi}^{2}\right)\left(m_{0}^{2}+2 m_{0} m_{D}+m_{D}^{2}-M_{\phi}^{2}\right)^{2} \\
& \times\left[5 m_{0}^{4} m_{D}^{(2)}-2 m_{0}^{2}\left(5 M_{\phi}^{2} m_{D}^{(2)}+m_{D}^{2}\left(m_{D}^{(2)}-m_{B}^{(2)}\right)\right)-2 m_{0}^{3} m_{D}\left(m_{D}^{(2)}+4 m_{B}^{(2)}\right)\right. \\
& \left.+2 m_{0} m_{D}\left(m_{D}^{2}+M_{\phi}^{2}\right)\left(m_{D}^{(2)}+4 m_{B}^{(2)}\right)-\left(m_{D}^{2}-M_{\phi}^{2}\right)\left(5 M_{\phi}^{2} m_{D}^{(2)}+m_{D}^{2}\left(3 m_{D}^{(2)}+2 m_{B}^{(2)}\right)\right)\right] \\
& \times\left[\arctan \left(\frac{m_{0}^{2}-m_{D}^{2}-M_{\phi}^{2}}{\sqrt{\mathcal{W}}}\right)-\arctan \left(\frac{m_{0}^{2}+m_{D}^{2}-M_{\phi}^{2}}{\sqrt{\mathcal{W}}}\right)\right] \text {, }
\end{aligned}
$$

$$
\begin{aligned}
& H_{D, D^{\prime}}^{(g)}\left(M_{\phi}\right)=\frac{M_{\phi}^{2}}{432 m_{D}^{4}}\left[4 m_{D}^{4}\left(132 m_{D}^{(2)}-97 m_{D^{\prime}}^{(2)}\right)\right. \\
& \left.+30 M_{\phi}^{4}\left(3 m_{D}^{(2)}+2 m_{D^{\prime}}^{(2)}\right)-15 m_{D}^{2} M_{\phi}^{2}\left(31 m_{D}^{(2)}+14 m_{D^{\prime}}^{(2)}\right)\right] \\
& +\frac{5 M_{\phi}^{2}}{72 m_{D}^{6}} \ln \left(\frac{M_{\phi}}{m_{D}}\right)\left[-30 m_{D}^{4} M_{\phi}^{2} m_{D}^{(2)}+48 m_{D}^{6}\left(m_{D}^{(2)}-m_{D^{\prime}}^{(2)}\right)\right. \\
& \left.+10 m_{D^{2}}^{2} M_{\phi}^{4}\left(2 m_{D}^{(2)}+m_{D^{\prime}}^{(2)}\right)-M_{\phi}^{6}\left(3 m_{D}^{(2)}+2 m_{D^{\prime}}^{(2)}\right)\right] \\
& +\frac{5 M_{\phi}^{2}}{36}\left(12 m_{D}^{(2)}-7 m_{D^{\prime}}^{(2)}\right) \ln \left(\frac{m_{D}^{2}}{\mu^{2}}\right) \\
& -\frac{5}{72 m_{D}^{6}} M_{\phi}^{3}\left(4 m_{D}^{2}-M_{\phi}^{2}\right)^{3 / 2}\left[2 m_{D}^{2}\left(m_{D}^{(2)}-m_{D^{\prime}}^{(2)}\right)-M_{\phi}^{2}\left(3 m_{D}^{(2)}+2 m_{D^{\prime}}^{(2)}\right)\right] \\
& \times\left[\arctan \left(\frac{M_{\phi}}{\sqrt{4 m_{D}^{2}-M_{\phi}^{2}}}\right)+\arctan \left(\frac{2 m_{D}^{2}-M_{\phi}^{2}}{M_{\phi} \sqrt{4 m_{D}^{2}-M_{\phi}^{2}}}\right)\right] .
\end{aligned}
$$


TABLE VII. Coefficients of the NLO contributions to the octet baryon masses [Eq. (36)].

\begin{tabular}{|c|c|c|c|c|}
\hline & $N$ & $\Lambda$ & $\Sigma$ & $\Xi$ \\
\hline$\xi_{B, \pi}^{(a)}$ & $-\left(2 b_{0}+4 b_{F}\right)$ & $\frac{-2}{3}\left(3 b_{0}-2 b_{D}\right)$ & $-\left(2 b_{0}+4 b_{D}\right)$ & $-\left(2 b_{0}-4 b_{F}\right)$ \\
\hline$\xi_{B, K}^{(a)}$ & $-\left(4 b_{0}+4 b_{D}-4 b_{F}\right)$ & $\frac{-2}{3}\left(6 b_{0}+8 b_{D}\right)$ & $-4 b_{0}$ & $-\left(4 b_{0}+4 b_{D}+4 b_{F}\right)$ \\
\hline
\end{tabular}

In Eqs. (34) and (35), $\mathcal{W}=-m_{0}^{4}-\left(m_{D}^{2}-M_{\phi}^{2}\right)^{2}+2 m_{0}^{2}\left(m_{D}^{2}+M_{\phi}^{2}\right), m_{D}^{(2)}$ and $m_{B}^{(2)}$ are the NLO decuplet and octet baryon masses, where $m_{D}^{(2)}$ is given in Eq. (13), and $m_{B}^{(2)}$ has the following form:

$$
m_{B}^{(2)}=\sum_{\phi=\pi, K} \xi_{B, \phi}^{(2)} M_{\phi}^{2}
$$

with the corresponding coefficients $\xi_{B, \phi}^{(2)}$ listed in Table VII.

[1] S. Durr, Z. Fodor, J. Frison, C. Hoelbling, R. Hoffmann, et al., Science 322, 1224 (2008), arXiv:0906.3599 [hep-lat].

[2] C. Alexandrou et al. (ETM Collaboration), Phys.Rev. D80, 114503 (2009) arXiv:0910.2419 [hep-lat].

[3] S. Aoki et al. (PACS-CS Collaboration), Phys.Rev. D79, 034503 (2009), arXiv:0807.1661 [hep-lat]

[4] S. Aoki et al. (PACS-CS Collaboration), Phys.Rev. D81, 074503 (2010), arXiv:0911.2561 [hep-lat].

[5] A. Walker-Loud, H.-W. Lin, D. Richards, R. Edwards, M. Engelhardt, et al., Phys.Rev. D79, 054502 (2009), arXiv:0806.4549 [hep-lat].

[6] H.-W. Lin et al. (Hadron Spectrum Collaboration), Phys.Rev. D79, 034502 (2009). arXiv:0810.3588 [hep-lat]

[7] W. Bietenholz, V. Bornyakov, N. Cundy, M. Gockeler, R. Horsley, et al., Phys.Lett. B690, 436 (2010). arXiv:1003.1114 [hep-lat].

[8] W. Bietenholz, V. Bornyakov, M. Gockeler, R. Horsley, W. Lockhart, et al., Phys.Rev. D84, 054509 (2011), arXiv:1102.5300 [hep-lat].

[9] S. Beane, E. Chang, W. Detmold, H. Lin, T. Luu, et al., Phys.Rev. D84, 014507 (2011). arXiv:1104.4101 [hep-lat].

[10] Z. Fodor and C. Hoelbling, Rev.Mod.Phys. 84, 449 (2012), arXiv:1203.4789 [hep-lat].

[11] J. Beringer et al. (Particle Data Group), Phys.Rev. D86, 010001 (2012). 
[12] S. Durr, Z. Fodor, C. Hoelbling, S. Katz, S. Krieg, et al., JHEP 1108, 148 (2011), arXiv:1011.2711 [hep-lat].

[13] A. Bazavov et al. (MILC Collaboration), Phys.Rev. D87, 054505 (2013), arXiv:1212.4768 [hep-lat].

[14] A. Bazavov et al. (MILC Collaboration), Phys.Rev.Lett. 110, 172003 (2013) arXiv:1301.5855 [hep-ph].

[15] S. Weinberg, Physica A96, 327 (1979).

[16] J. Gasser and H. Leutwyler, Annals Phys. 158, 142 (1984)

[17] J. Gasser and H. Leutwyler, Nucl.Phys. B250, 465 (1985)

[18] J. Gasser, M. Sainio, and A. Svarc, Nucl.Phys. B307, 779 (1988).

[19] H. Leutwyler, (1994), arXiv:hep-ph/9406283 [hep-ph]

[20] V. Bernard, N. Kaiser, and U.-G. Meissner, Int.J.Mod.Phys. E4, 193 (1995) arXiv:hep-ph/9501384 [hep-ph].

[21] A. Pich, Rept.Prog.Phys. 58, 563 (1995), arXiv:hep-ph/9502366 [hep-ph]

[22] G. Ecker, Prog.Part.Nucl.Phys. 35, 1 (1995), arXiv:hep-ph/9501357 [hep-ph].

[23] A. Pich, (1998), arXiv:hep-ph/9806303 [hep-ph]

[24] V. Bernard and U.-G. Meissner, Ann.Rev.Nucl.Part.Sci. 57, 33 (2007) arXiv:hep-ph/0611231 [hep-ph].

[25] V. Bernard, Prog.Part.Nucl.Phys. 60, 82 (2008), arXiv:0706.0312 [hep-ph].

[26] S. Scherer and M. R. Schindler, Lect.Notes Phys. 830, 1 (2012).

[27] D. B. Leinweber, A. W. Thomas, and R. D. Young, Phys.Rev.Lett. 92, 242002 (2004). arXiv:hep-lat/0302020 [hep-lat].

[28] V. Bernard, T. R. Hemmert, and U.-G. Meissner, Nucl.Phys. A732, 149 (2004). arXiv:hep-ph/0307115 [hep-ph].

[29] M. Procura, T. R. Hemmert, and W. Weise, Phys.Rev. D69, 034505 (2004), arXiv:hep-lat/0309020 [hep-lat].

[30] V. Bernard, T. R. Hemmert, and U.-G. Meissner, Phys.Lett. B622, 141 (2005), arXiv:hep-lat/0503022 [hep-lat].

[31] A. Ali Khan et al. (QCDSF-UKQCD Collaboration), Nucl.Phys. B689, 175 (2004), arXiv:hep-lat/0312030 [hep-lat].

[32] S. R. Beane, Phys.Rev. D70, 034507 (2004), arXiv:hep-lat/0403015 [hep-lat].

[33] S. R. Beane and M. J. Savage, Phys.Rev. D68, 114502 (2003), arXiv:hep-lat/0306036 [hep-lat]. 
[34] D. Arndt and B. C. Tiburzi, Phys.Rev. D69, 114503 (2004), arXiv:hep-lat/0402029 [hep-lat].

[35] E. E. Jenkins and A. V. Manohar, Phys.Lett. B255, 558 (1991)

[36] T. Becher and H. Leutwyler, Eur.Phys.J. C9, 643 (1999), arXiv:hep-ph/9901384 [hep-ph]

[37] J. Gegelia and G. Japaridze, Phys.Rev. D60, 114038 (1999), arXiv:hep-ph/9908377 [hep-ph].

[38] T. Fuchs, J. Gegelia, G. Japaridze, and S. Scherer, Phys.Rev. D68, 056005 (2003). arXiv:hep-ph/0302117 [hep-ph].

[39] E. E. Jenkins, Nucl.Phys. B368, 190 (1992)

[40] V. Bernard, N. Kaiser, and U. G. Meissner, Z.Phys. C60, 111 (1993), arXiv:hep-ph/9303311 [hep-ph]

[41] M. Banerjee and J. Milana, Phys.Rev. D52, 6451 (1995), arXiv:hep-ph/9410398 [hep-ph]

[42] B. Borasoy and U.-G. Meissner, Annals Phys. 254, 192 (1997), arXiv:hep-ph/9607432 [hep-ph].

[43] A. Walker-Loud, Nucl.Phys. A747, 476 (2005), arXiv:hep-lat/0405007 [hep-lat].

[44] P. Ellis and K. Torikoshi, Phys.Rev. C61, 015205 (2000), arXiv:nucl-th/9904017 [nucl-th].

[45] M. Frink and U.-G. Meissner, JHEP 0407, 028 (2004), arXiv:hep-lat/0404018 [hep-lat].

[46] M. Frink, U.-G. Meissner, and I. Scheller, Eur.Phys.J. A24, 395 (2005). arXiv:hep-lat/0501024 [hep-lat]

[47] B. Lehnhart, J. Gegelia, and S. Scherer, J.Phys. G31, 89 (2005), arXiv:hep-ph/0412092 [hep-ph],

[48] A. Semke and M. Lutz, Nucl.Phys. A778, 153 (2006), arXiv:nucl-th/0511061 [nucl-th].

[49] A. Semke and M. Lutz, Nucl.Phys. A789, 251 (2007)

[50] J. Martin Camalich, L. S. Geng, and M. Vicente Vacas, Phys.Rev. D82, 074504 (2010), arXiv:1003.1929 [hep-lat].

[51] L. S. Geng, X.-L. Ren, J. Martin-Camalich, and W. Weise, Phys.Rev. D84, 074024 (2011), arXiv:1108.2231 [hep-ph]

[52] R. Young and A. Thomas, Phys.Rev. D81, 014503 (2010), arXiv:0901.3310 [hep-lat].

[53] A. Semke and M. Lutz, Phys.Rev. D85, 034001 (2012), arXiv:1111.0238 [hep-ph]

[54] A. Semke and M. Lutz, Phys.Lett. B717, 242 (2012), arXiv:1202.3556 [hep-ph],

[55] M. Lutz and A. Semke, Phys.Rev. D86, 091502 (2012), arXiv:1209.2791 [hep-lat].

[56] P. C. Bruns, L. Greil, and A. Schafer, Phys.Rev. D87, 054021 (2013), arXiv:1209.0980 [hep-ph].

[57] X.-L. Ren, L. Geng, J. Martin Camalich, J. Meng, and H. Toki, J. High Energy Phys. 12, 073 (2012), arXiv:1209.3641 [nucl-th]

[58] X.-L. Ren, L. Geng, J. Meng, and H. Toki, Phys.Rev. D87, 074001 (2013) arXiv:1302.1953 [nucl-th] 
[59] K.-I. Ishikawa et al. (PACS-CS Collaboration), Phys.Rev. D80, 054502 (2009). arXiv:0905.0962 [hep-lat].

[60] V. Pascalutsa, Phys.Rev. D58, 096002 (1998), arXiv:hep-ph/9802288 [hep-ph].

[61] V. Pascalutsa and R. Timmermans, Phys.Rev. C60, 042201 (1999), arXiv:nucl-th/9905065 [nucl-th].

[62] L. S. Geng, J. Martin Camalich, L. Alvarez-Ruso, and M. Vicente Vacas, Phys.Rev. D78, 014011 (2008), arXiv:0801.4495 [hep-ph],

[63] M. Lutz and A. Semke, Phys.Rev. D83, 034008 (2011), arXiv:1012.4365 [hep-ph]

[64] B. C. Tiburzi and A. Walker-Loud, Nucl.Phys. A748, 513 (2005), arXiv:hep-lat/0407030 [hep-lat].

[65] V. Pascalutsa and M. Vanderhaeghen, Phys.Lett. B636, 31 (2006) arXiv:hep-ph/0511261 [hep-ph].

[66] L. S. Geng, Front.Phys.China. 8, 328 (2013), arXiv:1301.6815 [nucl-th]

[67] J. Bijnens and I. Jemos, Nucl.Phys. B854, 631 (2012), arXiv:1103.5945 [hep-ph]

[68] V. Bernard, U.-G. Meissner, and A. Rusetsky, Nucl.Phys. B788, 1 (2008) arXiv:hep-lat/0702012 [HEP-LAT].

[69] X.-L. Ren, L.-S. Geng, and J. Meng, Eur.Phys.J. C74, 2754 (2014) arXiv:1311.7234 [hep-ph].

[70] J. Alarcon, L. Geng, J. Martin Camalich, and J. Oller, Phys.Lett. B730, 342 (2014), arXiv:1209.2870 [hep-ph].

[71] A. Walker-Loud, Phys.Rev. D86, 074509 (2012), arXiv:1112.2658 [hep-lat].

[72] J. F. Donoghue, B. R. Holstein, and B. Borasoy, Phys.Rev. D59, 036002 (1999), arXiv:hep-ph/9804281 [hep-ph].

[73] R. D. Young, D. B. Leinweber, and A. W. Thomas, Prog.Part.Nucl.Phys. 50, 399 (2003), arXiv:hep-lat/0212031 [hep-lat].

[74] S. Descotes-Genon, N. Fuchs, L. Girlanda, and J. Stern, Eur.Phys.J. C34, 201 (2004). arXiv:hep-ph/0311120 [hep-ph],

[75] V. Bernard, S. Descotes-Genon, and G. Toucas, JHEP 1101, 107 (2011), arXiv:1009.5066 [hep-ph],

[76] L. S. Geng, J. Martin Camalich, L. Alvarez-Ruso, and M. Vicente Vacas, Phys.Rev.Lett. 101, 222002 (2008), arXiv:0805.1419 [hep-ph]. 